\title{
The origin of the breathing mode in Hall thrusters and its stabilisation
}

T. Lafleur, ${ }^{1}$ P. Chabert, ${ }^{2}$ and A. Bourdon ${ }^{2}$

1) PlasmaPotential-Physics Consulting and Research, Canberra, ACT 2601, Australia $^{\text {a) }}$

2) Laboratoire de Physique des Plasmas, CNRS, Sorbonne Universités, UPMC Univ Paris 06, Univ Paris Sud, Ecole Polytechnique, F-91128 Palaiseau, France

(Dated: 4 July 2021)

Using both $0 \mathrm{D}$ and $1 \mathrm{D}$ fluid models, we revisit the formation of the breathing mode in Hall thrusters and show that it is an ionization instability associated with nonlinearity in the electron power absorption. As the plasma density increases, the axial electric field profile changes and the magnitude of the electric field is enhanced in the ionization zone. This causes a nonlinear increase in the power absorbed by electrons, and an increase in the electron temperature and ionization rate factor that is able to partially compensate for the decreasing neutral density to keep the ionization rate high. This sets up a positive feedback mechanism where the electric field continues to be enhanced as the plasma density increases, and consequently the neutral density needs to decrease even further before plasma growth can be halted. At this point the neutral density is so low that the plasma can no longer be "sustained", and time is needed for neutrals to refill the thruster channel before "re-ignition" can occur and the process repeated. By treating the breathing mode as an AC excitation, a carefully designed external circuit can be used to counteract the change in axial electric field by appropriately varying the anode voltage to stabilise the discharge.

Keywords: Hall thruster, breathing mode, predator-prey, ionization instability

\footnotetext{
a)Electronic mail: trevor.lafleur@plasmapotential.com.au. Current affiliation: ThrustMe, 91370 Verrièresle-Buisson, France.
} 


\section{INTRODUCTION}

Hall-effect thrusters (HTs) are well-known to exhibit a large number of plasma oscillations spanning the $\mathrm{kHz}$ and $\mathrm{MHz}$ ranges ${ }^{1,2}$. Of these oscillations, the breathing mode (BM) is perhaps the most prominent $^{3}$, and is frequently observed in fluid simulations ${ }^{4,5}$, particle-incell (PIC) simulations ${ }^{6}$, and experiments ${ }^{7-11}$. This mode has a frequency in the $\mathrm{kHz}$ range (typically of the order of $10 \mathrm{kHz}$ ) and is often associated with a predator-prey type instability where the neutral gas plays the role of the "prey", and the plasma is the "predator" 12 . The BM leads to oscillations in the discharge current and almost all plasma properties ${ }^{3,4}$, and affects overall thruster performance.

Although the BM has been observed for more than 50 years, the exact cause of this phenomenon is still not well established. One of the first models of the BM was proposed by Fife ${ }^{12}$, who treated only the neutral and ion continuity equations in a $0 \mathrm{D}$ model with a constant ionization rate factor. A first-order perturbation analysis of the equations then leads to a harmonic oscillator response with a frequency in the $\mathrm{kHz}$ range. In this model the $\mathrm{BM}$ is a simple predator-prey interaction, and this is still widely regarded as the primary cause of these low-frequency oscillations ${ }^{3}$. However, subsequent analyses ${ }^{13,14}$ have pointed out one main assumption used in this model that is incorrect: the injected neutral mass flow rate is not considered, and when included, has the effect of leading to a system of equations that is unconditionally stable (i.e. no BM can develop). Additionally, since the model is $0 \mathrm{D}$, the length used to discretise the spatial convection terms is not well defined, and it is unclear if this length should be the thruster channel length, or the length of the ionization zone.

In the predator-prey interpretation of the BM, the oscillation frequency is determined by the local ionization dynamics, which suggests that this phenomenon should be observable in a $0 \mathrm{D}$ model. A second interpretation of the BM instead relates the oscillations to the convective motion of the neutral gas $^{4,13}$, and the time scale of the BM is associated not with local ionization dynamics, but rather with the time needed for neutrals to refill the ionization zone $e^{4}$, which is of the order of the neutral gas transit time. This is the interpretation originally proposed by Boeuf and Garrigues ${ }^{4}$, where a back and forth motion of the neutrals was observed in their 1D simulations, and which led to referencing of these types of oscillations as breathing. 
Subsequent studies have extended the original analysis by Fife ${ }^{12}$, by including, for example, perturbations in the electron current ${ }^{15}$, perturbations in the electron temperature ${ }^{14,16}$, the effect of the electron temperature evolution and thruster boundary conditions ${ }^{17}$, and more advanced theoretical approaches that appear to assume a form for the discharge current $^{5,18}$. Hara ${ }^{14}$ revisited the BM problem, and performing a linear perturbation analysis, argued that a positive growth rate is only obtained if perturbations in the electron energy equation are included. However, subsequent studies have argued that the instability criteria obtained may be unphysical ${ }^{19}$. The BM has also more recently been studied theoretically and experimentally by Dale and Jorns ${ }^{20,21}$, who assessed a number of possibilities as to the theoretical cause of the BM, including kinetic processes, spatial variations, and the presence of an additional energy source driving the instability. Their results indicate that a $0 \mathrm{D}$ model may not be sufficient to capture the system dynamics, and a two-zone model was proposed that couples the near-anode and ionization regions. In particular, fluctuations in the neutral density (due to ionization near the anode) entering the ionization zone may lead to the growth of an instability with frequencies similar to those observed for the BM. Although some agreement with experiment was observed ${ }^{21}$, the authors noted that it is not clear if ionization in the near-anode region is sufficient enough to modulate the neutral flow.

In this work we relook at the BM in HTs to better understand the cause of this phenomenon, and in particular, what triggers the formation of these low-frequency oscillations. To do this we start with a 0D model. As will be presented below, no BM is observed in 0D and the equations are stable. This leads to a 1D simulation model where finally the BM is produced. We then reduce complexity in this $1 \mathrm{D}$ model until the trigger for the BM is isolated. Finally, we discuss how the BM can be stabilised by making use of an external circuit.

\section{OD THRUSTER MODEL}

\section{A. Model equations}

To analyse the BM, we begin with a 0D model based on similar equations to those used previously in Ref. ${ }^{14}$

$$
\frac{d n_{g}}{d t}+\frac{\left(n_{g}-n_{g, i n j}\right) v_{g}}{L_{c h}}=-n_{e} n_{g} K_{i z}
$$




$$
\begin{gathered}
\frac{d n_{i}}{d t}+\frac{n_{i} v_{i}}{L_{c h}}+\frac{2 n_{i} u_{B}}{\Delta r}=n_{e} n_{g} K_{i z} \\
\frac{d}{d t}\left(m_{i} n_{i} v_{i}\right)+\frac{m_{i} n_{i} v_{i}^{2}}{L_{c h}}+\frac{2 m_{i} n_{i} u_{B} v_{i}}{\Delta r}=e n_{i} E \\
\frac{d}{d t}\left(\frac{3}{2} n_{e} T_{e}\right)+\frac{5}{2} \frac{n_{e} v_{e} T_{e}}{L}=-n_{e} v_{e} E-n_{e} \nu_{w} \varepsilon_{w}-n_{e} n_{g} K_{i z} \varepsilon_{i z} \chi
\end{gathered}
$$

where $t$ is time, $n_{g}$ is the neutral gas density, $n_{g, i n j}$ is the injected neutral gas density at the anode, $v_{g}$ is the neutral gas drift velocity (assumed constant), $L_{c h}$ is the length of the HT channel, $n_{e}$ is the electron density, $K_{i z}$ is the ionization rate factor, $n_{i}$ is the ion density, $v_{i}$ is the ion drift velocity, $u_{B}=\sqrt{\frac{e T_{e}}{m_{i}}}$ is the Bohm velocity with $e$ the elementary charge (assuming singly-charged positive ions) and $m_{i}$ the ion mass, $\Delta r$ is the width of the HT channel, $E=\frac{\phi_{d}}{L}$ is the accelerating electric field with $\phi_{d}$ the applied discharge voltage and $L$ the effective length of the ionization zone (see below), $T_{e}$ is the electron temperature (in units of $[\mathrm{eV}]), v_{e}$ is the electron drift velocity, $\varepsilon_{i z}$ is the ionization threshold of the neutral gas, $\chi$ is an effective ionization cost factor accounting for excitation losses, and the ionization rate factor, wall collision frequency, secondary electron emission coefficient due to electron bombardment, and electron kinetic energy losses at the wall, are given by

$$
\begin{gathered}
K_{i z}=\left(-1.024 \times 10^{-24} T_{e}^{2}+6.386 \times 10^{-20} e^{-\varepsilon_{i z} / T_{e}}\right) \bar{v}_{e} \\
\nu_{w}=\frac{1}{\Delta r} \frac{u_{B}}{1-\sigma} \\
\sigma=\min \left(\sigma_{\max }, \frac{T_{e}}{25}\right) \\
\varepsilon_{w}=2 T_{e}+(1-\sigma) T_{e} \ln \left[(1-\sigma) \sqrt{\frac{m_{i}}{2 \pi m_{e}}}\right]
\end{gathered}
$$

with $\bar{v}_{e}=\sqrt{\frac{8 e T_{e}}{\pi m_{e}}}, \sigma_{\max }=0.986$ (see Ref. ${ }^{5,22}$ ), and $m_{e}$ the electron mass. Following Ref. ${ }^{14}$, the effective length of the ionization zone, $L$, can be defined from

$$
\frac{L}{L_{c h}}=\frac{1}{1+\frac{2 u_{B}}{v_{i}} \frac{L_{c h}}{\Delta r}}
$$

in which case Eqs. 2-3 simplify to

$$
\begin{gathered}
\frac{d n_{i}}{d t}+\frac{n_{i} v_{i}}{L}=n_{e} n_{g} K_{i z} \\
\frac{d}{d t}\left(m_{i} n_{i} v_{i}\right)+\frac{m_{i} n_{i} v_{i}^{2}}{L}=e n_{i} E
\end{gathered}
$$


The plasma is quasi-neutral so that $n_{e}=n_{i}$. Strictly speaking, an additional term should appear in Eq. 1 representing ion recombination on the radial walls. Furthermore, there is an ambiguity in what length should be used in the definition of $E$ and in Eq. 4 (i.e. $L_{c h}$ or $L)$. However, different tests performed during the linear perturbation analysis and direct numerical integration described below showed no significant differences, so for consistency we follow the conventions used in Ref. ${ }^{14}$.

\section{B. Linear perturbation analysis}

In the simplest possible model, the ion drift velocity and electron temperature are fixed in time, in which case only Eqs. 1 and 10 apply. A linear perturbation analysis is then performed by setting, for example, $n_{i}=n_{i 0}+\delta n_{i}$, and assuming that $\delta n_{i}$ has a time variation proportional to $e^{-i \omega t}$, where $\omega=\omega_{R}+i \gamma$ is a complex angular frequency. The steady-state solution of Eqs. 1 and 10 is

$$
\begin{gathered}
n_{g 0}=\frac{v_{i}}{K_{i z} L} \\
n_{i 0}=\left(n_{g, i n j}-\frac{v_{i}}{K_{i z} L}\right) \frac{v_{g} L}{v_{i} L_{c h}}
\end{gathered}
$$

while the first-order perturbed solution is

$$
\begin{gathered}
\omega_{R}= \pm \sqrt{n_{i 0} n_{g 0} K_{i z}^{2}-\gamma^{2}} \\
\gamma=-\frac{1}{2}\left(\frac{n_{g, i n j}}{n_{g, i n j}-n_{g 0}}\right) n_{i 0} K_{i z}
\end{gathered}
$$

These solutions are identical to those obtained previously ${ }^{14}$. Since the growth rate in Eq. 15 is always negative, the system is unconditionally stable and no BM is formed. By now allowing the ion drift velocity and electron temperature to vary by also considering the ion momentum conservation equation (Eq. 11), and the electron energy equation (Eq. 4), the steady-state solution is modified to

$$
\begin{gathered}
v_{i 0}=\sqrt{\frac{e \phi_{d}}{m_{i}}} \\
n_{g 0}=\frac{1}{K_{i z 0} L} \sqrt{\frac{e \phi_{d}}{m_{i}}} \\
n_{i 0}=\left(n_{g, i n j}-\frac{1}{K_{i z 0} L} \sqrt{\frac{e \phi_{d}}{m_{i}}}\right) \frac{v_{g} L}{L_{c h}} \sqrt{\frac{m_{i}}{e \phi_{d}}}
\end{gathered}
$$




$$
v_{e}=-\frac{\left(\nu_{w 0} \varepsilon_{w 0}+n_{g 0} K_{i z 0} \varepsilon_{i z} \chi\right) L}{\phi_{d}+\frac{5}{2} T_{e 0}}
$$

and the new first-order perturbed solution in matrix form is

$$
\left[\begin{array}{cccc}
-i \omega & -n_{i 0} K_{i z} & \frac{n_{i 0}}{L} & -\left.n_{i 0} n_{g 0} \frac{d K_{i z}}{d T_{e}}\right|_{T_{e 0}} \\
\frac{v_{i 0}}{L} & -i \omega+\left(\frac{n_{g, i n j}}{n_{g, i n j}-n_{g 0}}\right) n_{i 0} K_{i z} & 0 & \left.n_{i 0} n_{g 0} \frac{d K_{i z}}{d T_{e}}\right|_{T_{e 0}} \\
-i \omega v_{i 0} & 0 & n_{i 0}\left(-i \omega+\frac{2 v_{i 0}}{L}\right) & 0 \\
-i \omega \frac{3}{2} T_{e 0} & n_{i 0} K_{i z 0} \varepsilon_{i z} \chi & 0 & n_{i 0}\left(-i \frac{3}{2} \omega+\Lambda\right)
\end{array}\right]\left[\begin{array}{c}
\delta n_{i} \\
\delta n_{g} \\
\delta v_{i} \\
\delta T_{e}
\end{array}\right]=0
$$

where

$$
\Lambda=\frac{5}{2} \frac{v_{e}}{L}+\frac{3}{2} \frac{\varepsilon_{w 0}}{T_{e 0}} \nu_{w 0}+\left.n_{g 0} \frac{d K_{i z}}{d T_{e}}\right|_{T_{e 0}} \varepsilon_{i z} \chi
$$

In the above equations, for convenience, we have used $T_{e 0}$ as a parameter, and consequently the steady-state electron energy equation (Eq. 19) determines the electron drift velocity. The variables, $\nu_{w a l l 0}, \varepsilon_{\text {wall } 0}$, and $K_{i z 0}$ are evaluated at $T_{e 0}$, and first-order perturbations in $L, \sigma$ and $\chi$ have been ignored. The determinant of the matrix equation above is analytically cumbersome, so is solved numerically instead. Solutions are shown in Fig. 1 as a function of the electron temperature for $L_{c h}=2.5 \mathrm{~cm}, \Delta r=2 \mathrm{~cm}, v_{g}=200 \mathrm{~ms}^{-1}, \phi_{d}=300 \mathrm{~V}$, and $n_{g, i n j}=1.6 \times 10^{19} \mathrm{~m}^{-3}$. For $T_{e 0}<15 \mathrm{eV}$, ionization is insufficient to sustain the discharge. The sharp change in slope at $T_{e 0}=25 \mathrm{eV}$ occurs because of the discontinuous change in $\sigma$ that occurs for $T_{e 0}>25 \mathrm{eV}$. All solutions have a negative growth rate and are again stable so that no BM forms. Because the determinant of the matrix equation must be solved numerically, it is difficult to prove that the system of equations is unconditionally stable for all operating conditions. However, we have performed a wide range of additional numerical tests with $10 \leq T_{e} \leq 30 \mathrm{eV}, 1 \leq L_{c h} \leq 6 \mathrm{~cm}, 1 \leq \Delta r \leq 6 \mathrm{~cm}, 100 \leq v_{g} \leq 600$ $\mathrm{ms}^{-1}, 100 \leq \phi_{d} \leq 600 \mathrm{~V}$, and $10^{19} \leq n_{g} \leq 10^{21} \mathrm{~m}^{-3}$, and for operating conditions leading to physically realisable discharges (where the plasma density is positive for example), the growth rate is always found to be negative.

Despite using the same model, and arriving at the same matrix equation, the conclusion obtained above is the opposite to that in Ref. ${ }^{14}$. Our results are however similar to the more recent analysis in Ref. ${ }^{19}$, where using a similar 0D model it was found that the system of equations was incapable of producing an instability, and the growth rate was always negative: even if the temporal variation of the electron temperature was accounted for. The authors found that the instability criteria in Ref. ${ }^{14}$ leading to a positive growth rate were 
unphysical, and that it appears the BM cannot be predicted using a conventional 0D model.
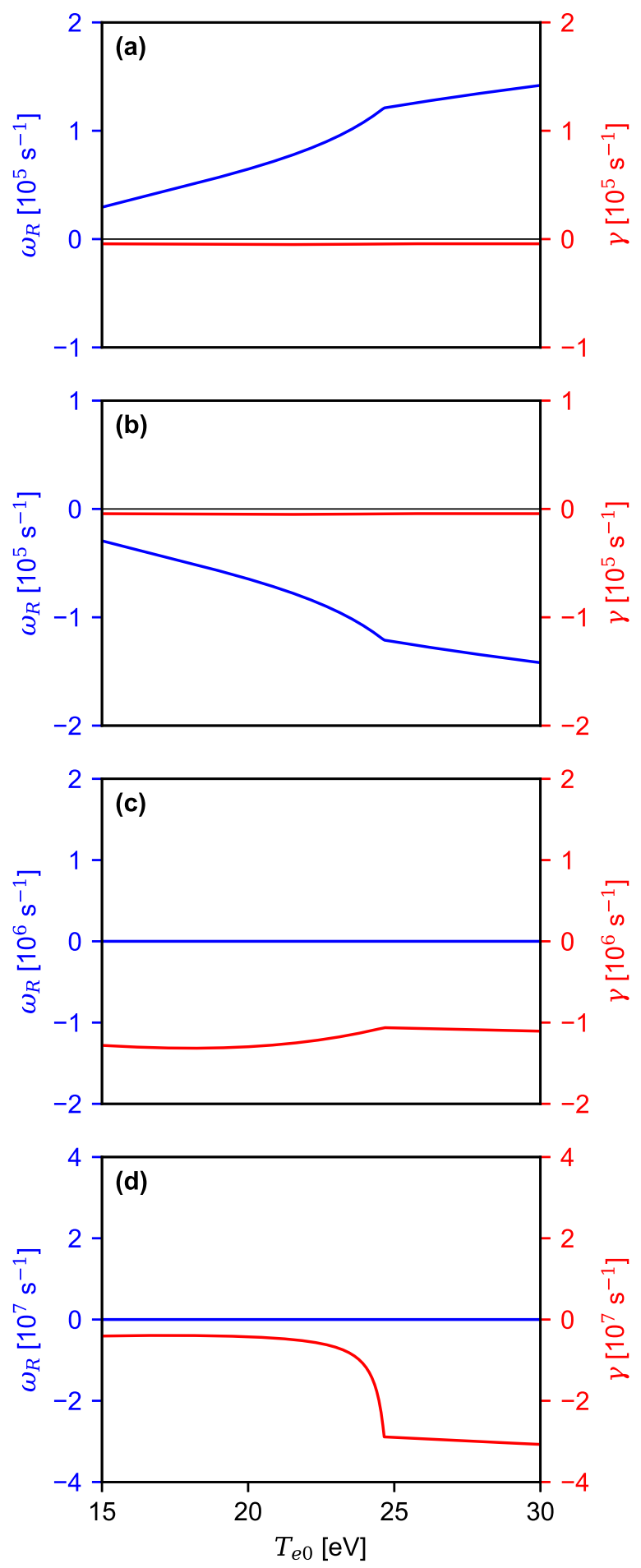

FIG. 1. The real angular frequency and growth rate for each root [(a)-(d)] of the determinant. 


\section{Direct numerical integration}

To check the results of the linear perturbation analysis in Section II B above, a direct numerical integration of Eqs. 1-4 is performed. Equations are solved using a 4th order Runge-Kutta scheme, and example results for $n_{g}(t=0)=1.6 \times 10^{19} \mathrm{~m}^{-3}, n_{i}(t=0)=$ $1 \times 10^{10} \mathrm{~m}^{-3}, v_{i}(t=0)=v_{g}$, and $T_{e}(t=0)=20 \mathrm{eV}$, are shown in Fig. 2. An initial transient oscillation is present with a frequency in the $\mathrm{kHz}$ range, but this oscillation is damped and eventually vanishes. The final steady-state solutions of the plasma properties match those predicted theoretically from Eqs. 16-19. Similar results are observed for different initial and operating conditions. Thus, direct numerical integration confirms the linear perturbation analysis.
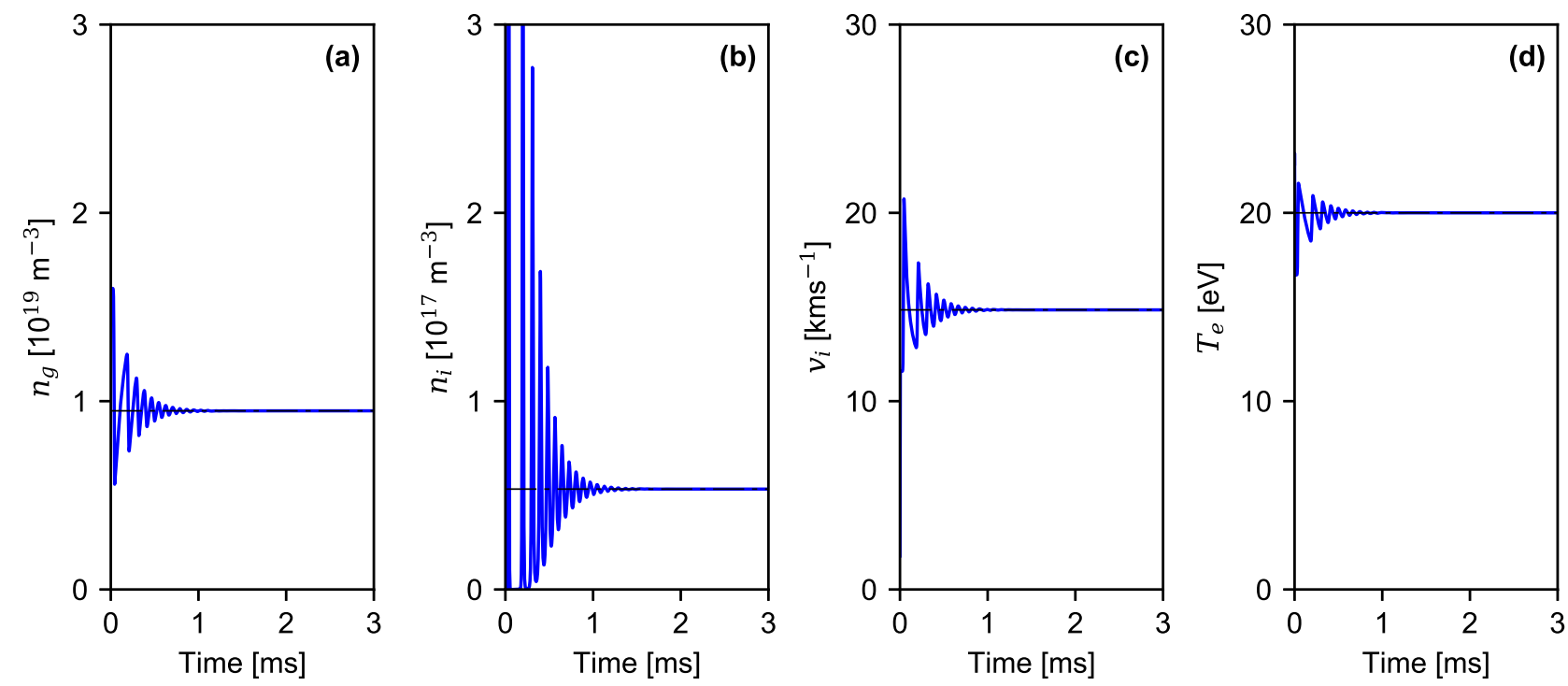

FIG. 2. Evolution of the (a) neutral gas density, $n_{g}$, (b) ion density, $n_{i}$, (c) ion drift velocity, $v_{i}$, and (d) electron temperature, $T_{e}$, in time. The horizontal black dash-dot lines show the steady-state solutions from Eqs. 16-19. 


\section{1D THRUSTER MODEL}

\section{A. Model equations}

Since no BM is observed with the 0D model, we proceed to a 1D model (similar to that used in Ref. ${ }^{5}$ ), and consisting of the following equations

$$
\begin{gathered}
\frac{\partial n_{g}}{\partial t}+v_{g} \frac{\partial n_{g}}{\partial x}=-n_{e} n_{g} K_{i z}+n_{i} \nu_{i w} \\
\frac{\partial n_{i}}{\partial t}+\frac{\partial}{\partial x}\left(n_{i} v_{i}\right)=n_{e} n_{g} K_{i z}-n_{i} \nu_{i w} \\
\frac{\partial}{\partial t}\left(m_{i} n_{i} v_{i}\right)+\frac{\partial}{\partial x}\left(m_{i} n_{i} v_{i}^{2}\right)=e n_{i} E_{x}+m_{i} n_{i} n_{g} K_{i z} v_{g}-m_{i} n_{i} \nu_{i w} v_{i} \\
\frac{\partial}{\partial t}\left(\frac{3}{2} n_{e} T_{e}\right)+\frac{\partial}{\partial x}\left(\frac{5}{2} n_{e} v_{e x} T_{e}\right)=-n_{e} v_{e x} E_{x}-n_{e} \nu_{e w} \varepsilon_{w}-n_{e} n_{g} K_{i z} \varepsilon_{i z} \chi
\end{gathered}
$$

These are the 1D extensions of Eqs. 1-4, with extra terms accounting for ion recombination on the radial channel walls, and ion momentum due to ionization. In addition, the following electron momentum conservation equations are added

$$
\begin{gathered}
0=-e n_{e} E_{x}-e n_{e} v_{e y} B_{z}-e \frac{\partial}{\partial x}\left(n_{e} T_{e}\right)-m_{e} n_{e} \nu_{m} v_{e x} \\
0=e n_{e} v_{e x} B_{z}-m_{e} n_{e} \nu_{m} v_{e y}
\end{gathered}
$$

In the equations above, $\nu_{i w}$ is the effective ion-wall collision frequency, $E_{x}=-\frac{d \phi}{d x}$ is the axial electric field with $\phi$ the electric potential, $v_{e x}$ and $v_{e y}$ are the "axial" and "azimuthal" electron drift velocities, $\nu_{e w}$ is the effective electron-wall collision frequency, $B_{z}$ is the applied "radial" magnetic field, and $\nu_{m}$ is the effective momentum transfer collision frequency. The different collision frequencies are given by

$$
\begin{gathered}
\nu_{i w}=\frac{4}{3} \frac{u_{B}}{\Delta r} \\
\nu_{e w}=\frac{\nu_{i w}}{1-\sigma} \\
\nu_{m}=n_{g} K_{e l}+\nu_{e w}+\alpha_{B} \omega_{c e}
\end{gathered}
$$

Here $K_{e l}=2.5 \times 10^{-13} \mathrm{~m}^{3} \mathrm{~s}^{-1}$ is the electron-neutral elastic momentum transfer rate factor, and the last term in Eq. 29 is a simple phenomenological model accounting for anomalous electron cross-field transport with $\alpha_{B}=6.25 \times 10^{-3}$, and $\omega_{c e}=\frac{e B_{z}}{m_{e}}$ the electron cyclotron frequency ${ }^{5}$. All other variables in the equations above have the same definition as those 
in Section II, except for the ionization rate factor, which is evaluated using the modified expression

$$
K_{i z}=1.8 \times 10^{-13}\left(\frac{3 T_{e}}{2 \varepsilon_{i z}}\right)^{1 / 4} e^{-\frac{4 \varepsilon_{i z}}{3 T_{e}}}
$$

Combining Eqs. 25 and 26, we obtain an expression for the axial electron drift velocity

$$
v_{e x}=-\mu_{e f f}\left[E_{x}+\frac{1}{n_{e}} \frac{\partial}{\partial x}\left(n_{e} T_{e}\right)\right]
$$

where

$$
\mu_{e f f}=\frac{e}{m_{e} \nu_{m}} \frac{1}{1+\left(\frac{\omega_{c e}}{\nu_{m}}\right)^{2}}
$$

The plasma discharge is again assumed to be quasi-neutral, and hence $n_{e}=n_{i}$. In order to model an external circuit connected to the HT (discussed in more detail later in Section IV), we consider an RL circuit, as illustrated in Fig. 3.

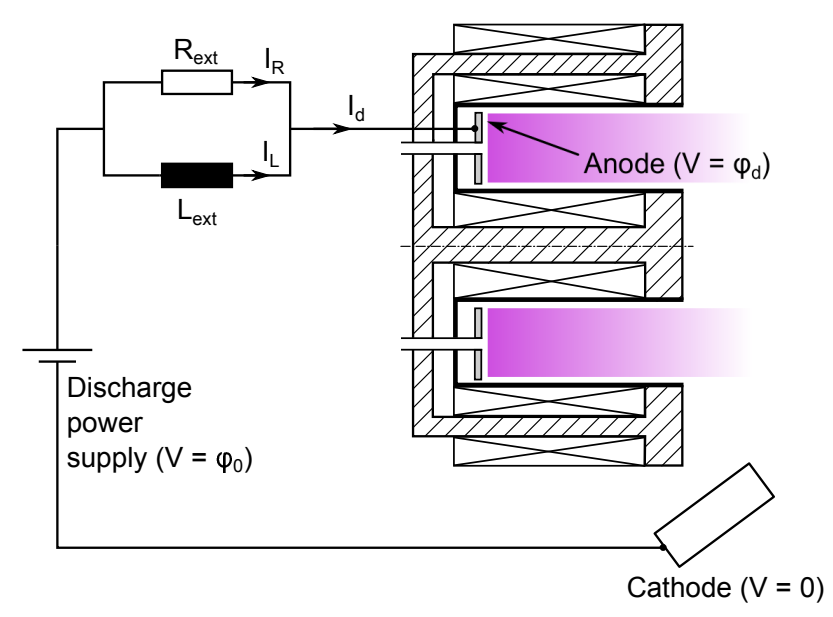

FIG. 3. Schematic of a Hall thruster with an external RL circuit.

The relevant circuit equations are

$$
\begin{gathered}
I_{R} R_{e x t}=L_{e x t} \frac{d I_{L}}{d t} \\
I_{d}=I_{R}+I_{L} \\
\phi_{d}=\phi_{0}-I_{R} R_{e x t}
\end{gathered}
$$

Here $I_{R}$ and $I_{L}$ are the currents flowing through the resistor and inductor respectively, $R_{e x t}$ is the resistance, $L_{\text {ext }}$ is the inductance, $I_{d}$ is the discharge current, and $\phi_{0}$ is a constant voltage applied from a DC voltage supply. The discharge current is also given by the sum of the 
electron and ion conduction currents in the discharge (assuming a negligible displacement current)

$$
I_{d}=-e n_{e} v_{e x} A+e n_{i} v_{i} A
$$

with $A$ the cross-sectional area of the channel. Combining Eqs. 33-36, integrating over the axial simulation domain length, $L$, and simplifying, we obtain a single differential equation for the inductor current

$$
\frac{d I_{L}}{d t}=\frac{e A \phi_{0}+e A \int_{0}^{L} d x\left[\frac{v_{i}}{\mu_{e f f}}+\frac{1}{n_{i}} \frac{d}{d x}\left(n_{i} T_{e}\right)\right]-I_{L} \int_{0}^{L} d x \frac{1}{n_{i} \mu_{e f f}}}{e A L_{e x t}+\frac{L_{e x t}}{R_{e x t}} \int_{0}^{L} d x \frac{1}{n_{i} \mu_{e f f}}}
$$

Once the inductor current is known, the resistor current and discharge current are found from Eqs. 33 and 34. The electric field is determined by combining Eqs. 31 and 36 to obtain

$$
E_{x}=\frac{I_{d}-e n_{i} v_{i} A}{e n_{i} \mu_{e f f} A}-\frac{1}{n_{i}} \frac{d}{d x}\left(n_{i} T_{e}\right)
$$

Because of the spatial derivative in Eq. 38, numerical stability of the electron energy equation (Eq. 24) is improved by instead writing it in the following form

$$
\frac{\partial}{\partial t}\left(\frac{T_{e}^{3 / 2}}{n_{e}}\right)+v_{e x} \frac{\partial}{\partial x}\left(\frac{T_{e}^{3 / 2}}{n_{e}}\right)=\frac{T_{e}^{1 / 2}}{n_{e}^{2}}\left[\frac{\left(\Gamma_{d}-n_{i} v_{i}\right)^{2}}{n_{e} \mu_{e f f}}-n_{e} \nu_{e w} \varepsilon_{w}-n_{e} n_{g} K_{i z} \varepsilon_{i z} \chi-\frac{5}{2} n_{e} n_{g} K_{i z} T_{e}\right]
$$

where $\Gamma_{d}=\frac{I_{d}}{e A}$. Equation 39 is obtained by substituting Eq. 38 into Eq. 24, using Eq. 22, and simplifying. In Eq. 39, the first term in parentheses on the right-hand side approximately represents the electron power absorption density

$$
p_{a b s} \approx n_{e} \mu_{e f f} E_{x}^{2} \approx \frac{\left(\Gamma_{d}-n_{i} v_{i}\right)^{2}}{n_{e} \mu_{e f f}}
$$

The boundary conditions used for the above model are as follows: $\phi(x=0)=\phi_{d}, \phi(x=$ $L)=0, v_{i}(x=0)=-\sqrt{\frac{5 e T_{e}(x=0)}{3 m_{i}}}$, and $T_{e}(x=L)=5 \mathrm{eV}$. At the anode, we explicitly test the importance of ion recombination (which may be important in the formation of the $\mathrm{BM}^{17}$ ) by using the boundary condition, $n_{g}(x=0)=\frac{\dot{m}}{m_{i} v_{g} A}$ if ion recombination is neglected, and $n_{g}(x=0)=\frac{\dot{m}}{m_{i} v_{g} A}-\frac{n_{i}(x=0) v_{i}(x=0)}{v_{g}}$ if ion recombination is included. Here $\dot{m}$ is the input mass flow rate. The equations are solved using an explicit time integration method, and the spatial derivative for the neutrals and electron energy equation are solved using a first-order upwind difference scheme, while the spatial derivatives in the ion continuity and momentum conservation equation are treated using the HLL flux splitting scheme ${ }^{23}$, together with the 
modification proposed by Davis ${ }^{24}$. The spatial derivative of the electron pressure term is evaluated using a central difference scheme. Open boundary conditions are used at the last grid points where needed in the above numerical schemes. Finally, the magnetic field has the following form

$$
B_{z}=B_{0} e^{-\left(\frac{x-L_{c h}}{L_{B}}\right)^{2}}
$$

where $B_{0}=200 \mathrm{G}, L_{B}=\frac{L_{c h}}{2}$, and $L_{c h}=4 \mathrm{~cm}$. For all simulations, $L=4.5 \mathrm{~cm}, \Delta r=r_{2}-r_{1}$ where $r_{2}=5 \mathrm{~cm}$ and $r_{1}=3 \mathrm{~cm}, v_{g}=200 \mathrm{~ms}^{-1}, \dot{m}=5 \mathrm{mgs}^{-1}$, and $\chi=3$.

\section{B. Breathing mode oscillations}

We begin by first removing the RL circuit in Fig. 3, and neglecting ion recombination at the anode. In this case, $\phi_{d}=\phi_{0}$. Simulations are started with uniform spatial profiles of the neutral and ion density, ion velocity, and electron temperature, set to values of $2.28 \times 10^{19} \mathrm{~m}^{-3}, 1 \times 10^{15} \mathrm{~m}^{-3}, 0 \mathrm{~ms}^{-1}$, and $5 \mathrm{eV}$ respectively. An adaptive time step (with a typical value of the order of $1 \times 10^{-9} \mathrm{~s}$ ) is used, together with 100 spatial grid points. The time step is chosen to ensure that the Courant-Friedrichs-Lewy (CFL) condition is satisfied everywhere in the discharge. The time evolution of the discharge current is shown below in Fig. 4 for different values of the discharge voltage.
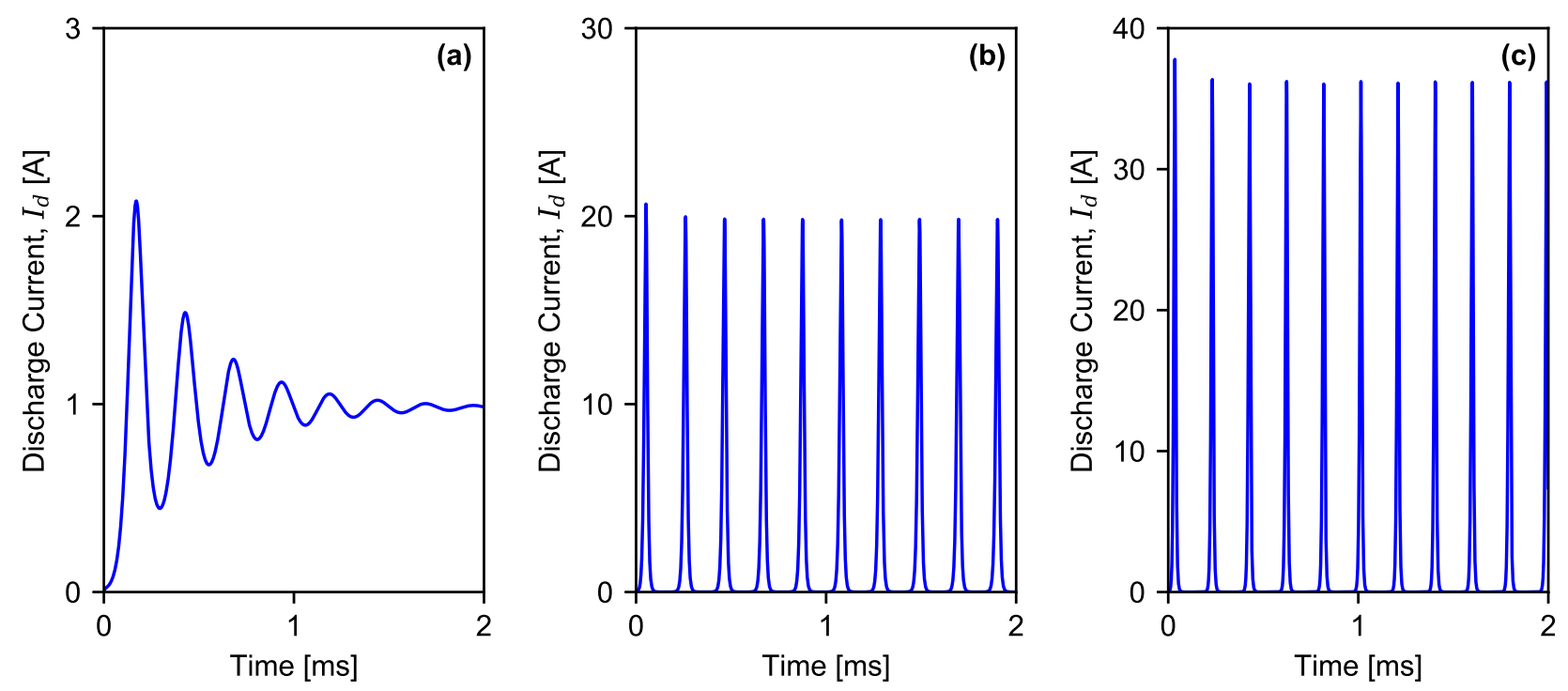

FIG. 4. Discharge current as a function of time for a discharge voltage of (a) $215 \mathrm{~V}$, (b) $250 \mathrm{~V}$, and (c) $275 \mathrm{~V}$. 
For discharge voltages above about $220 \mathrm{~V}$, a BM oscillation with a frequency of about 5 $\mathrm{kHz}$ is observed in the simulations; as demonstrated in Fig. 4 (b) and (c). For a voltage of $215 \mathrm{~V}$, a low-frequency oscillation is present, but eventually damps out until a steady-state is obtained. At higher voltages however, the BM persists. The amplitude of the discharge current peaks is also higher as the discharge voltage increases. These results are similar to those obtained in previous 1D fluid simulations of $\mathrm{HTs}^{4,5}$.

\section{Breathing mode trigger}

To find the trigger of the BM, a number of tests are performed. In these tests, simulations are run to equilibrium, and the external RL circuit is now included (with $R_{\text {ext }}=5 \Omega$ and $\left.L_{\text {ext }}=300 \mu \mathrm{H}\right)$. The effect of this circuit will be discussed further in Section IV, but by correctly choosing the value of the resistor and inductor, the BM can be stabilised and a stationary steady-state obtained. Once simulations have reached a stationary state, the RL circuit is removed and the system allowed to evolve further in time for four test cases: Case A where no change to the system of fluid equations is made, Case B where the ion drift velocity profile is fixed in time using the stationary state profile, Case $\mathbf{C}$ where both the ion drift velocity and the electron temperature profiles are fixed in time using the stationary state profiles, and Case D where the power absorption density (Eq. 40) is fixed in time using the stationary state profile. The results of these tests are shown below in Fig. 5 . 

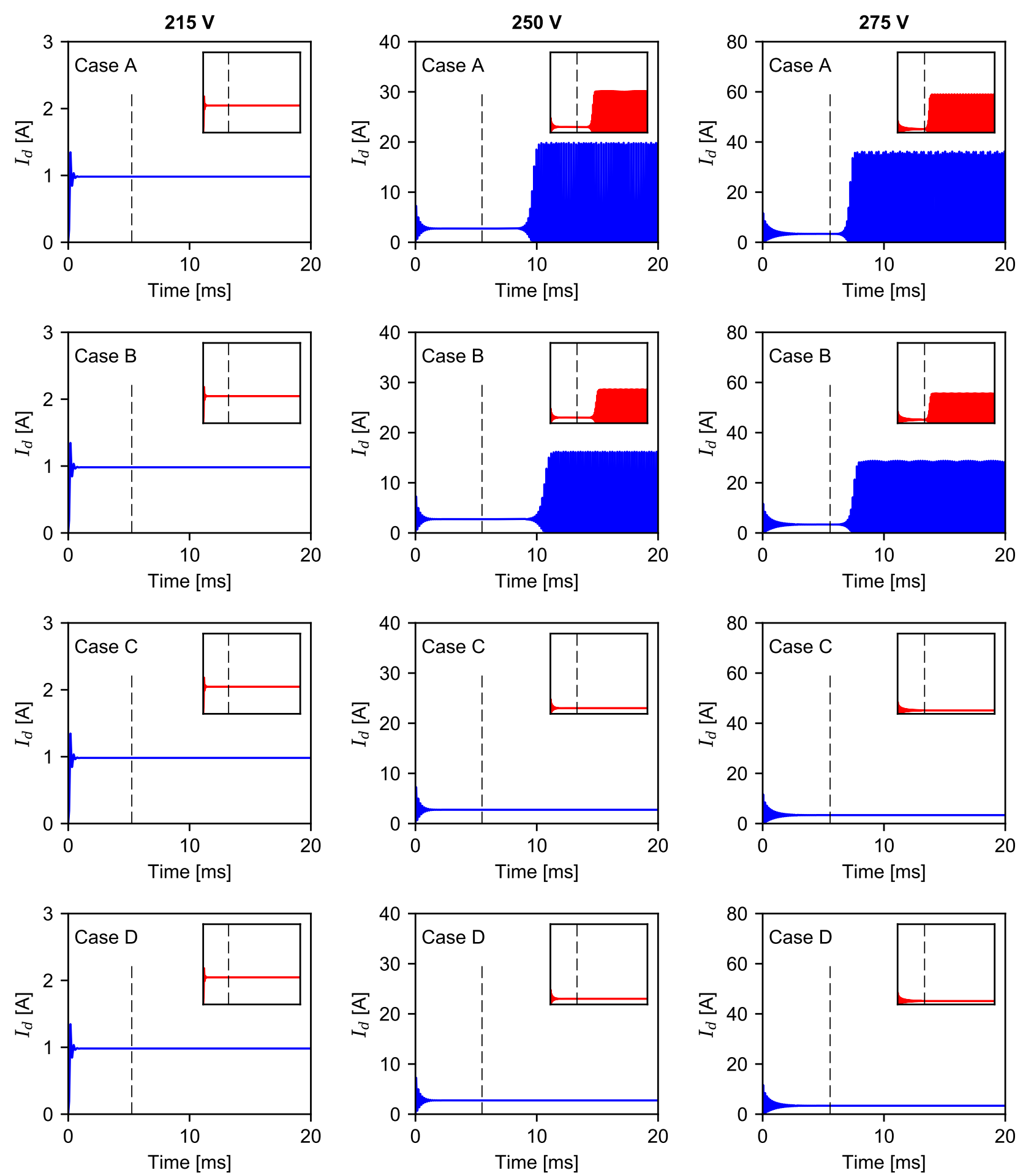

FIG. 5. Discharge current as a function of time. The columns denote different applied discharge voltages, while the rows denote the different test cases: Case A (no change to the plasma equations), Case B ( $v_{i}$ fixed), Case $\mathrm{C}\left(v_{i}\right.$ and $T_{e}$ fixed), and Case $\mathrm{D}$ ( $p_{a b s}$ fixed). The vertical black dashed lines denote the time at which the RL circuit was removed, and when each of the different test cases were activated. The inset figures show results when ion recombination at the anode is included. 
For $215 \mathrm{~V}$, once the system reaches a stationary state and the RL circuit is turned off, (indicated by the vertical black dashed line), all four test cases exhibit the same behaviour and this stationary state persists. By contrast, at the higher voltages of $250 \mathrm{~V}$ and $275 \mathrm{~V}$, different results are obtained depending on the test case. For Case A, after the RL circuit is turned off, an instability slowly develops until eventually, a low-frequency BM oscillation is again formed as in Fig. 4. This behaviour is similarly reproduced for Case B with a slight decrease in the discharge current amplitude. For Case $\mathbf{C}$, there is no observable change, and the original stationary state is maintained. Finally, for Case D, there is also no observable change.

Similar results (shown as the inset figures in Fig. 5) are obtained if ion recombination at the anode is included in the simulations. These results confirm that the primary cause of the BM is related to the electron temperature and electron power absorption, and that the BM can form even in the absence of ion recombination. The magnitude of the discharge current is however slightly higher when recombination is present, as is the growth rate of the BM instability (the large amplitude discharge current oscillations appear earlier in time after the RL circuit is turned off).

Despite the 1D simulation model being simplified, it still includes a number of important physical phenomena such as anomalous electron transport and wall losses. The anomalous transport collision frequency modifies the electron mobility and is important for allowing sufficient electron transport across the magnetic field when the neutral density is low. Therefore, turning this term off as an additional BM test case is challenging. However, since this term only affects the electron mobility, additional tests can be performed by fixing the mobility in time after the RL circuit is turned off (in addition to fixing the other profiles as in Cases A-D). Similar results to those in Fig. 5 (not shown) are again obtained. Thus, the mobility does not appear to be the primary trigger of the BM. Turning wall losses off makes the system of equations more unstable, and it is difficult to systemically repeat Cases A-D since the BM is found to be particularly violent. This is largely related to the electron-wall power loss term in Eq. 24, since by removing this term, the electron temperature can increase to higher values and hence enhance the ionization rate and resulting neutral depletion.

As an additional test (not shown in Fig. 5), the mobility and electric field in the electron power absorption density (Eq. 40) are fixed in time using their stationary state profiles, in which case the power absorption can now only vary linearly with plasma density. For 215 
$\mathrm{V}$, there is again no change observed and the stationary state persists after the RL circuit is removed. For $250 \mathrm{~V}$ and $275 \mathrm{~V}$, a brief transient develops before the system quickly evolves to a new, different, stationary state also with no oscillations. Thus, these results and those in Fig. 5 show that the BM is only formed if the electron temperature can vary in time, and more specifically, if the electric field changes so that the power absorption density changes nonlinearly. Observation of Eq. 40 shows that even if the mobility is fixed in time, this nonlinearity in the electron power absorption term remains.

Although the BM is present for both Case A and Case B in Fig. 5 for discharge voltages of $250 \mathrm{~V}$ and $275 \mathrm{~V}$, the amplitude and frequency spectrum of the discharge current is slightly different, as shown in Fig. 6. Here, the frequency spectrum shows a number of well-defined peaks centered on a fundamental frequency of about $5 \mathrm{kHz}$ and its higher harmonics. The amplitude of the fundamental frequency is slightly higher for Case $\mathbf{B}$, whereas most of the higher harmonics have a lower amplitude. There is also a small shift of the spectrum to higher frequencies for Case B. Thus, even though the ion drift velocity (the profile of which is kept constant for Case B) does not appear to be the primary cause of the BM, it nonetheless plays a role in its evolution once it forms.
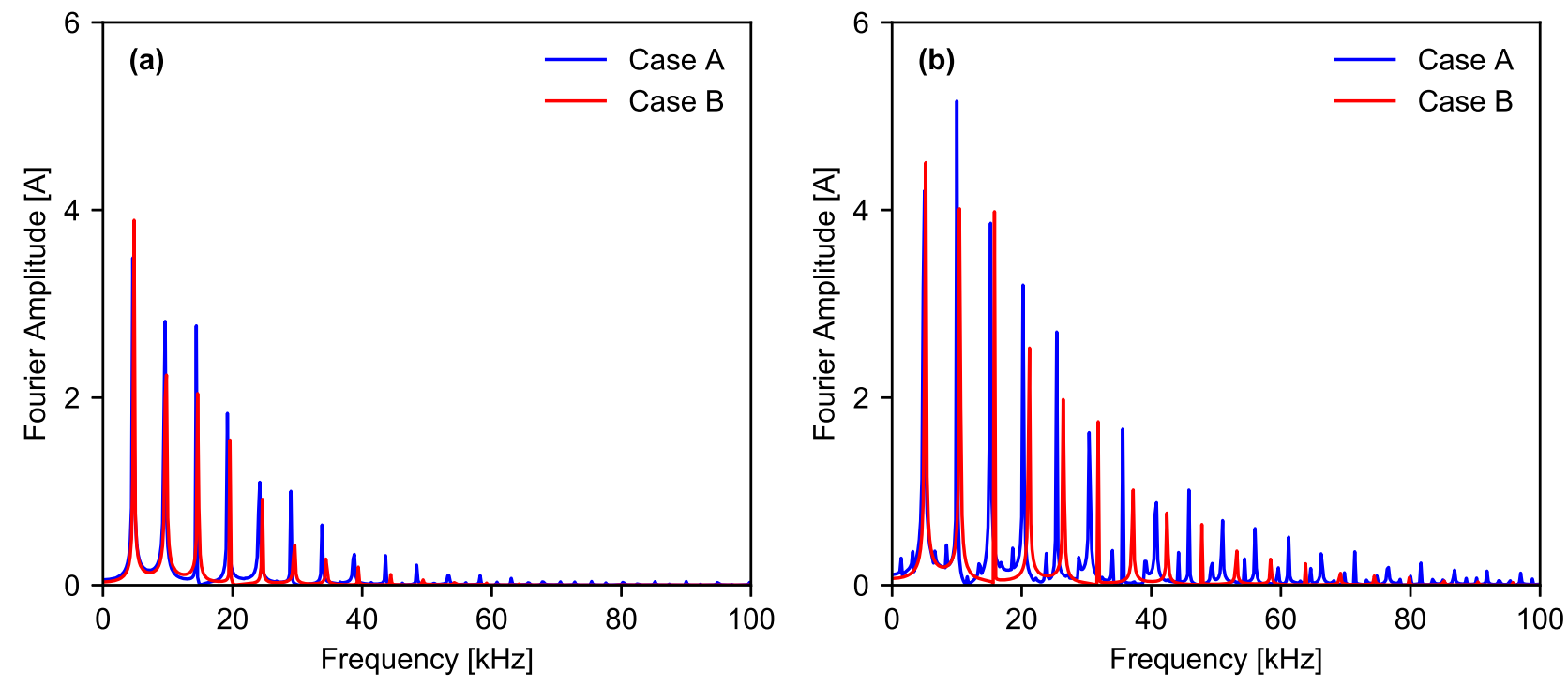

FIG. 6. Frequency spectrum of the discharge current for Case A and Case B in Fig. 5 for a discharge voltage of (a) $250 \mathrm{~V}$, and (b) $275 \mathrm{~V}$.

Further insight into the BM can be obtained by observing spatio-temporal plots of important plasma quantities as shown in Fig. 7 for $250 \mathrm{~V}$. The temporal reference (i.e. $t / T=0$ ) 
is taken at the time when the discharge current is a maximum. Initially, the neutral density profile is depleted, but as time proceeds, neutral re-filling occurs until the density starts to decrease again around $t / T=1$. Just before this time, the plasma density in Fig. 7 (b) is seen to rapidly increase. However, in addition, the electron temperature also increases (Fig. 7 (c)), and this leads to a corresponding increase in the ionization rate factor shown in Fig. 7 (d). Thus, even though the neutral density starts to decrease, both the plasma density and ionization rate factor increase, which allows the total ionization rate, $S_{i z}=n_{i} n_{g} K_{i z}$, to be higher than would be expected if the electron temperature were fixed in time. Eventually, the neutral density is depleted to such an extent that ionization can no longer be maintained, and the plasma density rapidly decreases. Because of this, the ionization rate becomes very low. This gives neutrals time to refill the channel, until eventually the density is high enough to once again allow sufficient ionization, and the cycle repeats. Thus, the $\mathrm{BM}$ is associated with a type of ionization instability.

A vital component of this instability is the temporal variation of the electric field, as illustrated in Fig. 8. Here, as the plasma density increases, the electric field is enhanced. However, from Eq. 40, if both the plasma density and electric field increase, this leads to a nonlinear increase in the electron power absorption. This produces a positive feedback loop whereby the increased electron power absorption allows the electron temperature and ionization rate factor to increase, which further increases the plasma density and electric field. This process continues until the neutral density is so low that sufficient ionization can no longer be sustained. If the power absorption density is fixed, as in Case $\mathbf{D}$, or if the electric field is fixed so that there is only a linear increase in power absorption with plasma density, the system can accommodate any changes and no positive feedback loop occurs.

Figure 9 shows the neutral density and ionization rate at three different BM cycle phases. In Fig. 9 (a), the ionization rate is low and the neutral density is high. This is the start of a discharge current pulse and the BM cycle. In 9 (b), the ionization rate has increased (because of a higher plasma density), while the neutral density begins to be depleted. Finally, Fig. 9 (c) shows a point in the BM cycle where significant neutral depletion has occurred. Despite this depletion, the ionization rate still remains high. If the electron temperature (and hence ionization rate factor) were constant in time, then as the neutral density gets depleted, the ionization rate decreases. However, because the electron temperature also changes, the ionization rate factor increases and now the ionization rate remains higher 

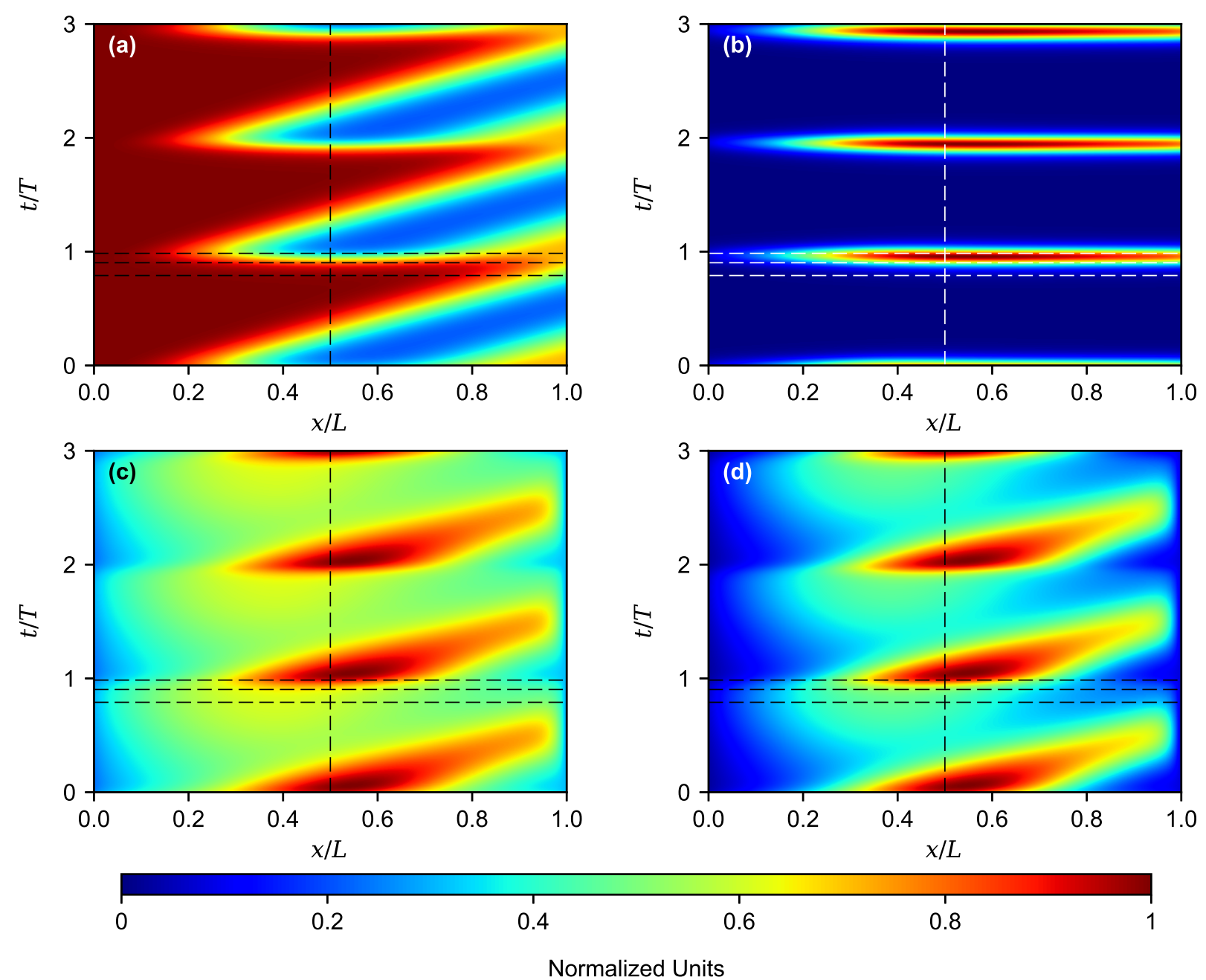

FIG. 7. Spatio-temporal plots of the normalized (a) neutral density, $n_{g}$, (b) plasma density, $n_{e}=n_{i}$, (c) electron temperature, $T_{e}$, and (d) ionization rate factor, $K_{i z}$, over $3 \mathrm{BM}$ cycles for Case B. The discharge voltage is $250 \mathrm{~V}$. The vertical black dashed lines indicate the discharge position used in Fig. 8, while the horizontal black dashed lines indicate the BM phase for the subplots in Fig. 9.

than it would otherwise be. This requires the neutral density to decrease even further before plasma growth is halted, and eventually, the neutral density decreases to such an extent that the plasma is almost completely extinguished. The neutrals then require some time to refill the channel before the plasma can be "reignited". The BM frequency for the above simulation conditions is about $5 \mathrm{kHz}$, which is similar to the transit time of neutrals through the simulation domain. 


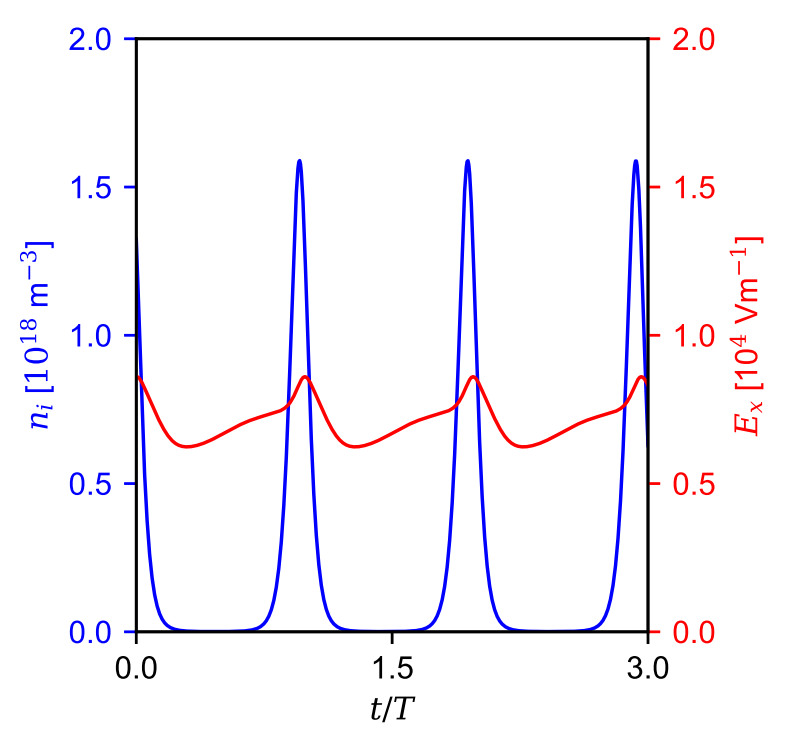

FIG. 8. Plasma density, $n_{e}=n_{i}$, and axial electric field, $E_{x}$, at $x / L=0.5$ as a function of time. The discharge voltage is $250 \mathrm{~V}$. 

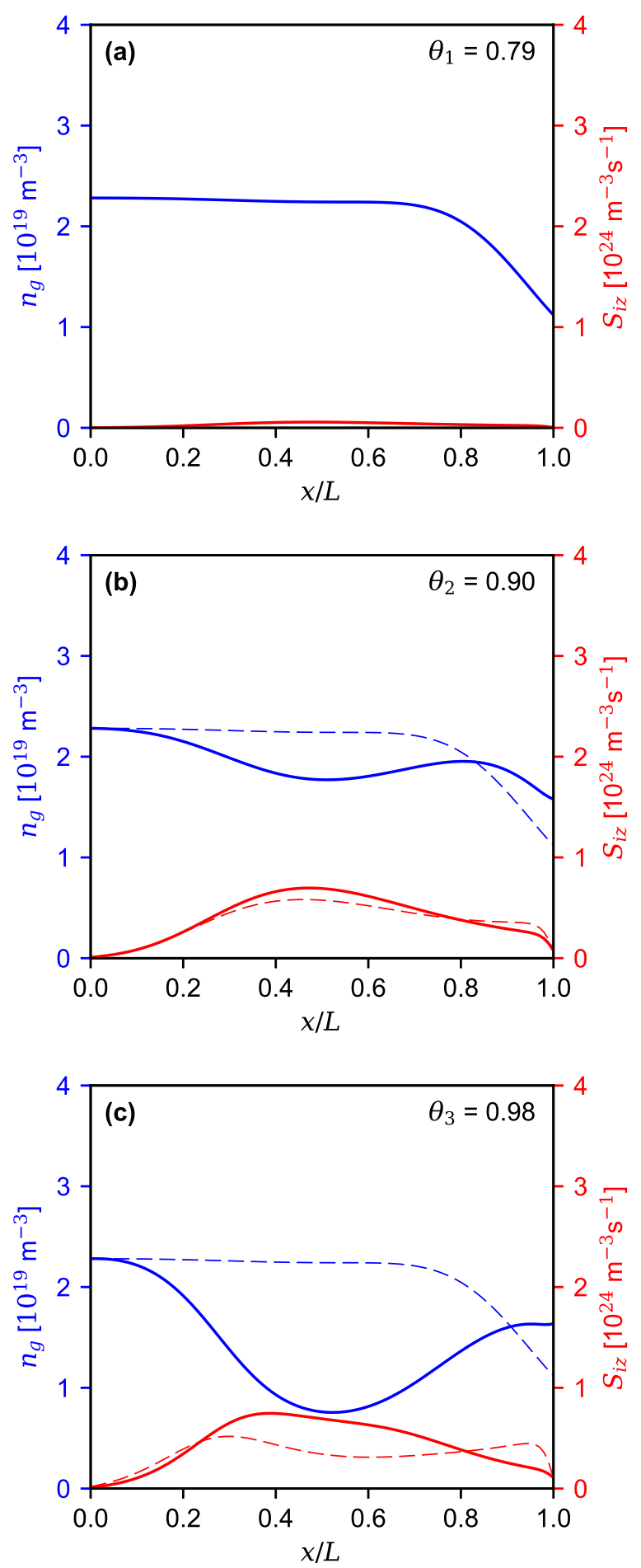

FIG. 9. Neutral density, $n_{g}$, and ionization rate, $S_{i z}=n_{g} n_{i} K_{i z}$, as a function of position for the three different BM phase positions indicated in Fig. 7: (a) $\theta_{1}=0.79$, (b) $\theta_{2}=0.90$, and (c) $\theta_{3}=0.98$, where $\left.\theta=t / T\right)$. For the middle and bottom subplots, the red dashed lines show the ionization rate if the electron temperature were fixed in time (using the values at $\theta_{1}$ ), while the blue dashed lines show the neutral density at $\theta_{1}$. The discharge voltage is $250 \mathrm{~V}$. 
The results above indicate that the origin of the BM is connected to nonlinearity within the electron power absorption term. Ignoring the RL circuit, the discharge current can be obtained from Eqs. 33-37

$$
\Gamma_{d}=\frac{\phi_{d}+\int_{0}^{L} d x\left[\frac{v_{i}}{\mu_{e f f}}+\frac{1}{n_{i}} \frac{d}{d x}\left(n_{i} T_{e}\right)\right]}{\int_{0}^{L} d x \frac{1}{n_{i} \mu_{e f f}}}
$$

Substituting this into Eq. 38, ignoring the electron pressure gradient terms, and simplifying, yields an approximate expression for the electric field

$$
E_{x} \approx \frac{\phi_{d}+\int_{0}^{L} d x \frac{v_{i}}{\mu_{e f f}}}{n_{i} \mu_{e f f} \int_{0}^{L} d x \frac{1}{n_{i} \mu_{e f f}}}-\frac{v_{i}}{\mu_{e f f}}
$$

This equation shows that the electric field is a function not only of the instantaneous local value of the plasma density (and also ion drift velocity and electron mobility), but also the global profile due to the presence of the integrals. In $0 \mathrm{D}$, there is no satisfactory way to account for spatial variations, and so the integrands are effectively assumed to be constant, giving $E_{x}=\phi_{d} / L$ which is independent of the plasma density, and always constant in time. Thus, from Eq. 40 the electron power absorption is essentially linear with plasma density. In 1D however, this is no longer the case, and the electric field can in general change giving a nonlinear variation of the electron power absorption. Since the electric field, and its spatiotemporal variation, depend on the applied voltage and magnetic field (through the electron mobility), one can expect that BM onset conditions exist: that is to say, the temporal variation of the axial electric field depends on the magnitude of the plasma density (for example), and so for sufficiently low discharge voltages, the variation may be low enough that nonlinearity in the electron power absorption is weak and no BM forms.

\section{CIRCUIT STABILISATION OF THE BREATHING MODE}

\section{A. AC circuit analogy}

The results in Section III show that the BM is linked to the electric field and electron power absorption. Since the electric field in Eq. 43 is a function of the discharge voltage, the BM may be stabilised if this discharge voltage is appropriately varied in time. External circuit filters have been widely used to reduce transient loads on the thruster 
power supply, and the coupled effects of the plasma discharge and power processing unit extensively studied since BM oscillations are observed to be very sensitive to power supply characteristics $^{1,8,25-29}$. The fundamental cause of the BM has historically been difficult to isolate due to this power supply coupling, and low-frequency oscillations have often been referred to as "loop", "circuit" or "contour" instabilities ${ }^{1,27}$. Another more recent stabilisation approach, used by Barral ${ }^{30}$, is a closed-loop feedback control mechanism using a proportional-integral-derivative controller applied to the discharge voltage to damp the BM. Here however, we consider only a passive external circuit filter, such as the RL circuit illustrated in Fig. 3. By then treating the $\mathrm{BM}$ as an $\mathrm{AC}$ excitation to this RL circuit, the complex impedance of the circuit can be controlled to change the phase shift or proportionally between the discharge current and voltage to damp the BM.

Considering the circuit in Fig. 3, if the BM exists, the discharge current can be viewed as consisting of a constant current component, and an $\mathrm{AC}$ current component. Considering this AC component as a simple sinusoidal excitation, the RL circuit has a complex impedance, $Z$, given by

$$
Z=\frac{\omega R L}{R^{2}+\omega^{2} L^{2}}(\omega L+j R)
$$

where $\omega$ is the angular frequency of the BM. The magnitude, $|Z|$, and phase, $\theta$, of the impedance are then easily found

$$
\begin{gathered}
|Z|=\frac{R}{\sqrt{1+\frac{R^{2}}{\omega^{2} L^{2}}}} \\
\tan \theta=\frac{R}{\omega L}
\end{gathered}
$$

The magnitude of the impedance determines the amplitude of the discharge voltage oscillations, while the phase determines the temporal shift between the discharge current and voltage oscillations. From this perspective, we see that the correct parameters to consider are not the resistance, $R$, and inductance, $L$, but rather $|Z|$ and $\theta$. For example, varying only $R$ while fixing $L$ (which is the approach used in some studies ${ }^{28,29}$ ) may not produce the desired circuit response, because both the magnitude and phase of the impedance are changed. Instead, the magnitude and argument should be chosen, and the value of the required resistance and inductance determined. By inverting Eqs. 45-46 we find

$$
R=\frac{|Z|}{\cos \theta}
$$




$$
L=\frac{|Z|}{\omega \sin \theta}
$$

For $0 \leq \theta \leq 90^{\circ}$.

\section{B. Breathing mode stabilisation}

By fixing the magnitude of the impedance, the BM can be stabilised by varying the impedance phase. The time evolution of the discharge current and voltage is shown below in Fig. 10 for an applied power supply voltage of $250 \mathrm{~V}$, and an impedance magnitude of $|Z|=5 \Omega$. For $\theta=10^{\circ}$ and $\theta=30^{\circ}$, the BM is strongly damped and a stationary state is obtained. As $\theta$ increases though, damping is reduced, and for sufficiently large values of $\theta$, the circuit no longer has any effect in stabilising the BM. The discharge voltage is also observed to oscillate. The amplitude of the discharge current peaks is however reduced compared to the case with no external circuit (see Fig. 4), and the peaks are less pronounced.

Figure 10 shows that by correctly selecting the circuit parameters, the BM can be stabilised. It should be noted that the BM in the $1 \mathrm{D}$ model can also be stabilised using just a resistor (placed in series with the HT). However, this is not a feasible solution for a real thruster since at steady-state, the full discharge current will flow through the resistor leading to large power losses, and a voltage drop across the resistor that reduces the effective discharge voltage.

\section{DISCUSSION AND CONCLUSIONS}

In summary, we have investigated the BM in HTs and found that this mode is associated with the axial electric field, and nonlinearity in the electron power absorption. In a $0 \mathrm{D}$ model, the axial electric field is always constant in time and electron power absorption is essentially linear with plasma density so that any changes in plasma density can easily be accommodated by the system to maintain stability. A linear perturbation analysis of the 0D HT equations gives a system that is stable, and this is confirmed by direct numerical integration. In 1D however, the spatial profile and magnitude of the axial electric field can now vary, and as the plasma density increases during a BM cycle, the electric field is enhanced in some regions of the discharge. This increases the electron power absorption, and leads to an increase in the electron temperature and ionization rate factor that partially 

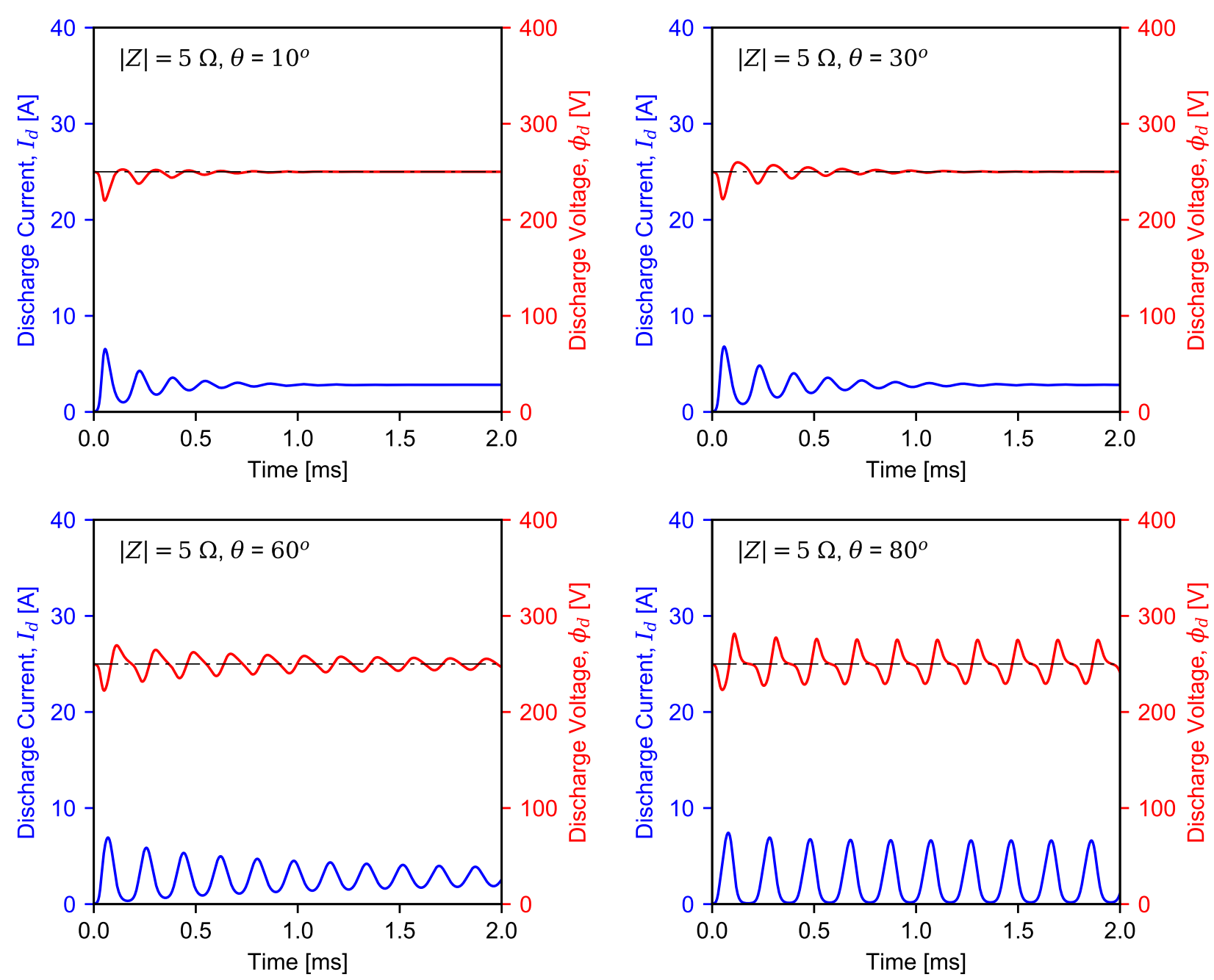

FIG. 10. Discharge current and voltage as a function of time for different impedance phases, $\theta$. The applied power supply voltage (horizontal black dash-dot lines) is $250 \mathrm{~V}$.

compensates for the decrease of total ionization rate due to the decreasing neutral density. Thus, initially, the decreasing neutral density is not sufficient to halt or stabilise plasma growth, and a positive feedback mechanism exists where as the plasma density increases, so too does the ionization rate factor. Eventually though, the neutral density decreases to such an extent that the plasma can no longer be "sustained", and the discharge current and plasma density rapidly decreases. However, because of the significant depletion that has now occurred, the neutral density is too low for the plasma to "reignite" and some time is required for neutrals to once again refill the channel.

Since the electron power absorption depends sensitively on the electric field, which is itself 
a function of the discharge voltage, the use of an external RL circuit represents one way in which the $\mathrm{BM}$ can be stabilised. By treating the BM as an $\mathrm{AC}$ excitation, and correctly designing the circuit to achieve a given complex impedance, the BM can be damped and a stationary state obtained. The idea here is to decrease the discharge voltage when the discharge current increases so as to reduce the electric field in such a way that the positive feedback mechanism can be broken. This stabilisation depends on the frequency of the BM, and the values of the resistance and inductance used, which cannot be treated as separate parameters as they both affect the impedance magnitude and phase. It should be noted that in the present analysis we have not included a capacitor in the external circuit. In any real HT, such a capacitor would likely be required in parallel with the thruster to act as a short-circuit for any high-frequency oscillations in the discharge current. The use of capacitive elements may also facilitate additional adjustment of the circuit impedance at different operating conditions (such as by allowing the phase to vary between $0^{\circ}$ and $360^{\circ}$ ). We also note that the focus of the present study has been on the basic physics of the BM, and while using an external circuit can lead to stabilisation, this may not always be desired. For example, the presence of the BM can be beneficial and lead to enhanced thruster performance ${ }^{31}$.

The 1D model we have used is a simplified representation of a HT, and makes a number of assumptions. Perhaps the most important is related to anomalous electron transport, which still remains an active area of research within the community ${ }^{32-41}$. No self-consistent first principles model for the cross-field electron transport currently exists, and so we have treated the transport using a simple ad hoc method. This method cannot be claimed to accurately represent the electron transport in any real HT, but it nonetheless reproduces many aspects of HT behaviour. Having a more sophisticated electron transport model is not expected to alter the main conclusions of this work, but it may well alter the ability of the external circuit to completely stabilise the BM. Since there is not yet any reliable anomalous electron transport model, further studies would be needed using either PIC simulations including an external circuit, or more preferably, direct experimental testing.

Finally, we note that depending on the 1D model used, there may be a second trigger leading to BM oscillations. In the results presented here, we have largely associated the BM with the electron power absorption term. However, from Eq. 43, the axial electric field is also a function of the ion drift velocity, and the ion momentum conservation equation 
depends on the axial electric field. Even if the electron temperature and ionization rate factor are fixed, nonlinearity in this ion-electric field acceleration term in the momentum conservation equation may also lead to oscillations under some conditions. Changes in the axial electric field can locally decrease the ion velocity and lead to a corresponding increase in the plasma density. This enhances the ionization rate, and may produce a new positive feedback mechanism. This hypothesis is confirmed by at least two simulation studies where low-frequency oscillations were observed when using 1D models with fixed electron temperatures and ionization rate factors (these assumptions are however unrealistic) ${ }^{26,28}$. The process though is still associated with variations in the axial electric field, which appear critical to the formation of the BM.

\section{DATA AVAILABILITY}

The data that support the findings of this study are available from the corresponding author upon reasonable request.

\section{REFERENCES}

${ }^{1}$ E. Choueiri, "Plasma oscillations in hall thrusters," Physics of Plasmas 8, 1411-1426 (2001).

2 J.-P. Boeuf, "Tutorial: Physics and modeling of hall thrusters," Journal of Applied Physics 121, 011101 (2017).

${ }^{3}$ D. M. Goebel and I. Katz, Fundamentals of electric propulsion: ion and Hall thrusters, Vol. 1 (John Wiley \&amp; Sons, 2008).

${ }^{4}$ J. Boeuf and L. Garrigues, "Low frequency oscillations in a stationary plasma thruster," Journal of Applied Physics 84, 3541-3554 (1998).

${ }^{5}$ S. Barral and E. Ahedo, "Low-frequency model of breathing oscillations in hall discharges," Physical Review E 79, 046401 (2009).

${ }^{6}$ J. Adam, A. Heron, and G. Laval, "Study of stationary plasma thrusters using twodimensional fully kinetic simulations," Physics of Plasmas 11, 295-305 (2004).

${ }^{7}$ Y. B. Esipchuk, A. Morozov, G. Tilinin, and A. Trofimov, "Plasma oscillations in closeddrift accelerators with an extended acceleration zone," Soviet Physics Technical Physics 
18, 928 (1974).

${ }^{8}$ G. Tilinin, "High-frequency plasma waves in a hall accelerator with an extended acceleration zone," Soviet Physics Technical Physics 22, 974-978 (1977).

${ }^{9}$ N. Gascon, M. Dudeck, and S. Barral, "Wall material effects in stationary plasma thrusters. i. parametric studies of an spt-100," Physics of Plasmas 10, 4123-4136 (2003).

${ }^{10} \mathrm{~V}$. Giannetti, M. Saravia, and T. Andreussi, "Measurement of the breathing mode oscillations in hall thruster plasmas with a fast-diving triple langmuir probe," Physics of Plasmas 27, 123502 (2020).

${ }^{11}$ C. Young, A. L. Fabris, N. MacDonald-Tenenbaum, W. Hargus, and M. Cappelli, "Timeresolved laser-induced fluorescence diagnostics for electric propulsion and their application to breathing mode dynamics," Plasma Sources Science and Technology 27, 094004 (2018).

${ }^{12}$ J. M. Fife, Hybrid-PIC modeling and electrostatic probe survey of Hall thrusters, Ph.D. thesis, Massachusetts Institute of Technology (1998).

${ }^{13} \mathrm{~S}$. Barral and E. Ahedo, "On the origin of low frequency oscillations in hall thrusters," in AIP Conference Proceedings, Vol. 993 (American Institute of Physics, 2008) pp. 439-442.

${ }^{14}$ K. Hara, M. J. Sekerak, I. D. Boyd, and A. D. Gallimore, "Perturbation analysis of ionization oscillations in hall effect thrusters," Physics of Plasmas 21, 122103 (2014).

${ }^{15}$ N. Yamamoto, K. Komurasaki, and Y. Arakawa, "Discharge current oscillation in hall thrusters," Journal of propulsion and power 21, 870-876 (2005).

${ }^{16} \mathrm{~S}$. Barral and Z. Peradzyński, "Ionization oscillations in hall accelerators," Physics of Plasmas 17, 014505 (2010).

${ }^{17}$ A. Smolyakov, O. Chapurin, I. Romadanov, Y. Raitses, and I. Kaganovich, "Theory and modelling of axial mode oscillations in hall thruster," (AIAA Propulsion and Energy 2019 Forum, AIAA 2019-4080, Indianapolis, Indiana, USA, 19-22 August 2019).

${ }^{18}$ Z. Peradzyński, K. Makowski, S. Barral, J. Kurzyna, and M. Dudeck, "The role of the electron energy balance in plasma thruster instabilities," in AIP Conference Proceedings, Vol. 993 (American Institute of Physics, 2008) pp. 435-438.

${ }^{19}$ E. T. Dale, B. Jorns, and K. Hara, "Numerical investigation of the stability criteria for the breathing mode in hall effect thrusters," (35th International Electric Propulsion Conference, IEPC 2017-265, Atlanta, Georgia, USA, 8-12 October 2017).

${ }^{20}$ E. T. Dale and B. A. Jorns, "Two-zone hall thruster breathing mode mechanism, part i: Theory," (36th International Electric Propulsion Conference, IEPC 2019-354, Vienna, 
Austria, 15-20 September 2019).

${ }^{21}$ E. T. Dale and B. A. Jorns, "Two-zone hall thruster breathing mode mechanism, part ii: Experiment," (36th International Electric Propulsion Conference, IEPC 2019-354, Vienna, Austria, 15-20 September 2019).

${ }^{22} \mathrm{G}$. Hobbs and J. Wesson, "Heat flow through a langmuir sheath in the presence of electron emission," Plasma Physics 9, 85 (1967).

${ }^{23}$ A. Harten, P. D. Lax, and B. v. Leer, "On upstream differencing and godunov-type schemes for hyperbolic conservation laws," SIAM review 25, 35-61 (1983).

${ }^{24}$ S. Davis, "Simplified second-order godunov-type methods," SIAM Journal on Scientific and Statistical Computing 9, 445-473 (1988).

${ }^{25}$ V. V. Zhurin, H. R. Kaufman, and R. S. Robinson, "Physics of closed drift thrusters," Plasma Sources Science and Technology 8, R1 (1999).

${ }^{26}$ A. Morozov and V. Savel'ev, "One-dimensional hybrid model of a stationary plasma thruster," Plasma Physics Reports 26, 875-880 (2000).

${ }^{27} \mathrm{~S}$. Barral, "Theoretical analysis of the influence of the power supply on breathing oscillations in hall thrusters," (30th International Electric Propulsion Conference, IEPC 2007-261, Florence, Italy, 17-20 September 2007).

${ }^{28}$ D. Yu, C. Wang, L. Wei, C. Gao, and G. Yu, "Stabilizing of low frequency oscillation in hall thrusters," Physics of Plasmas 15, 113503 (2008).

${ }^{29}$ W. Liqiu, W. Chunsheng, N. Zhongxi, L. Weiwei, Z. ChaoHai, and Y. Daren, "Experimental study on the role of a resistor in the filter of hall thrusters," Physics of Plasmas 18, 063508 (2011).

${ }^{30}$ S. Barral, J. Kaczmarczyk, J. Kurzyna, and M. Dudeck, "Closed-loop control of ionization oscillations in hall accelerators," Physics of Plasmas 18, 083504 (2011).

${ }^{31}$ J. Simmonds, Y. Raitses, A. I. Smolyakov, and O. Chapurin, "Studies of a modulated hall thruster," Plasma Sources Science and Technology (2021).

${ }^{32}$ I. D. Kaganovich, A. Smolyakov, Y. Raitses, E. Ahedo, I. G. Mikellides, B. Jorns, F. Taccogna, R. Gueroult, S. Tsikata, A. Bourdon, et al., "Physics of ex b discharges relevant to plasma propulsion and similar technologies," Physics of Plasmas 27, 120601 (2020).

${ }^{33}$ W. Frias, A. I. Smolyakov, I. D. Kaganovich, and Y. Raitses, "Long wavelength gradient drift instability in hall plasma devices. i. fluid theory," Physics of Plasmas 19, 072112 (2012). 
${ }^{34}$ J. Cavalier, N. Lemoine, G. Bonhomme, S. Tsikata, C. Honoré, and D. Grésillon, "Hall thruster plasma fluctuations identified as the $\mathrm{e} \times \mathrm{b}$ electron drift instability: Modeling and fitting on experimental data," Physics of Plasmas 20, 082107 (2013).

${ }^{35}$ S. Tsikata, N. Lemoine, V. Pisarev, and D. Grésillon, "Dispersion relations of electron density fluctuations in a hall thruster plasma, observed by collective light scattering," Physics of Plasmas 16, 033506 (2009).

${ }^{36}$ A. Héron and J. Adam, "Anomalous conductivity in hall thrusters: Effects of the nonlinear coupling of the electron-cyclotron drift instability with secondary electron emission of the walls," Physics of Plasmas 20, 082313 (2013).

${ }^{37}$ A. Ducrocq, J. Adam, A. Héron, and G. Laval, "High-frequency electron drift instability in the cross-field configuration of hall thrusters," Physics of Plasmas 13, 102111 (2006).

${ }^{38}$ T. Lafleur and P. Chabert, "The role of instability-enhanced friction on "anomalous" electron and ion transport in hall-effect thrusters", plasma sources science and technology," Plasma Sources Science and Technology 27 (2018).

${ }^{39}$ T. Lafleur, S. Baalrud, and P. Chabert, "Theory for the anomalous electron transport in hall effect thrusters. ii. kinetic model," Physics of Plasmas 23, 053503 (2016).

${ }^{40}$ I. Katz, A. Lopez Ortega, B. Jorns, and I. G. Mikellides, "Growth and saturation of ion acoustic waves in hall thrusters," (52nd AIAA/SAE/ASEE Joint Propulsion Conference, AIAA 2016-4534, Salt Lake City, Utah, USA, 25-27 July 2016).

${ }^{41}$ I. Katz, I. G. Mikellides, B. A. Jorns, and A. L. Ortega, "Hall2de simulations with an anomalous transport model based on the electron cyclotron drift instability," (34th International Electric Propulsion Conference, IEPC 2015-402, Kobe-Hyogo, Japan, 4-10 July 2015).

${ }^{42}$ K. Hara and I. G. Mikellides, "Characterization of low frequency ionization oscillations in hall thrusters using a one-dimensional fluid model," (2018 Joint Propulsion Conference, AIAA 2018-4904, Cincinnati, Ohio, USA, 9-11 July 2018).

${ }^{43} \mathrm{P}$. Coche and L. Garrigues, "A two-dimensional (azimuthal-axial) particle-in-cell model of a hall thruster," Physics of Plasmas 21, 023503 (2014). 


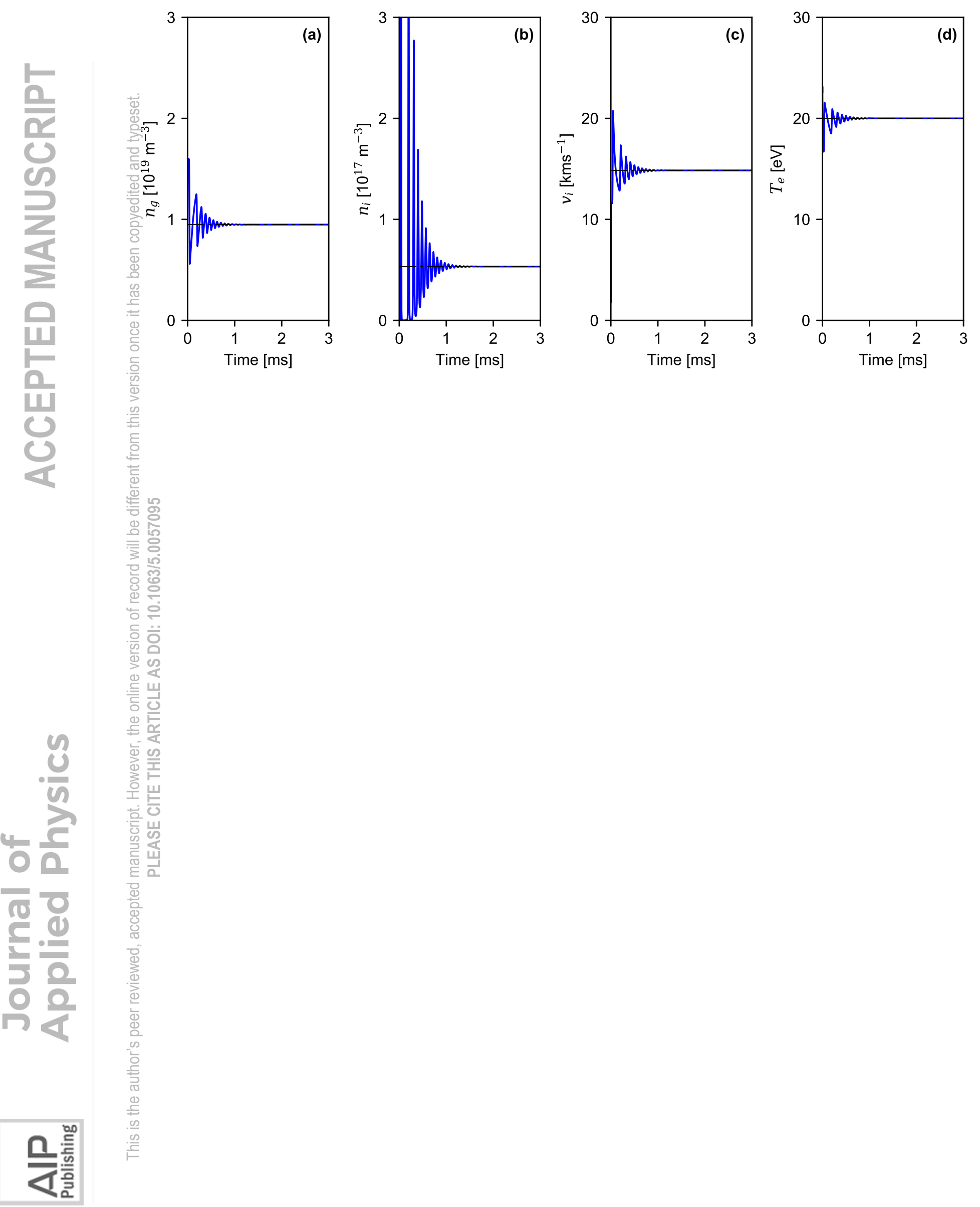




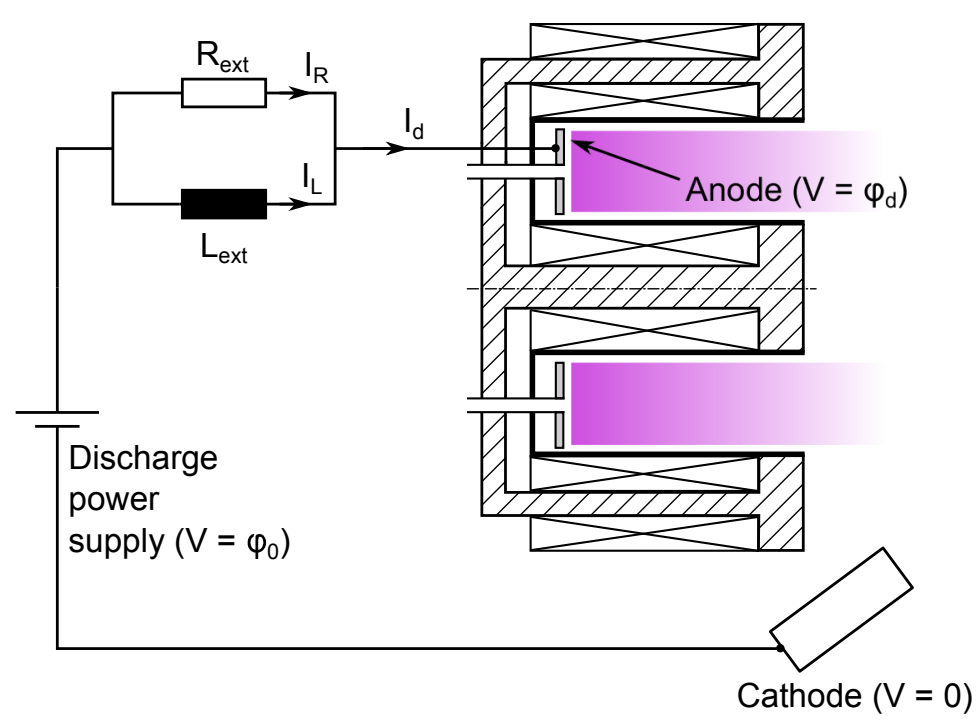


$215 \mathrm{~V}$
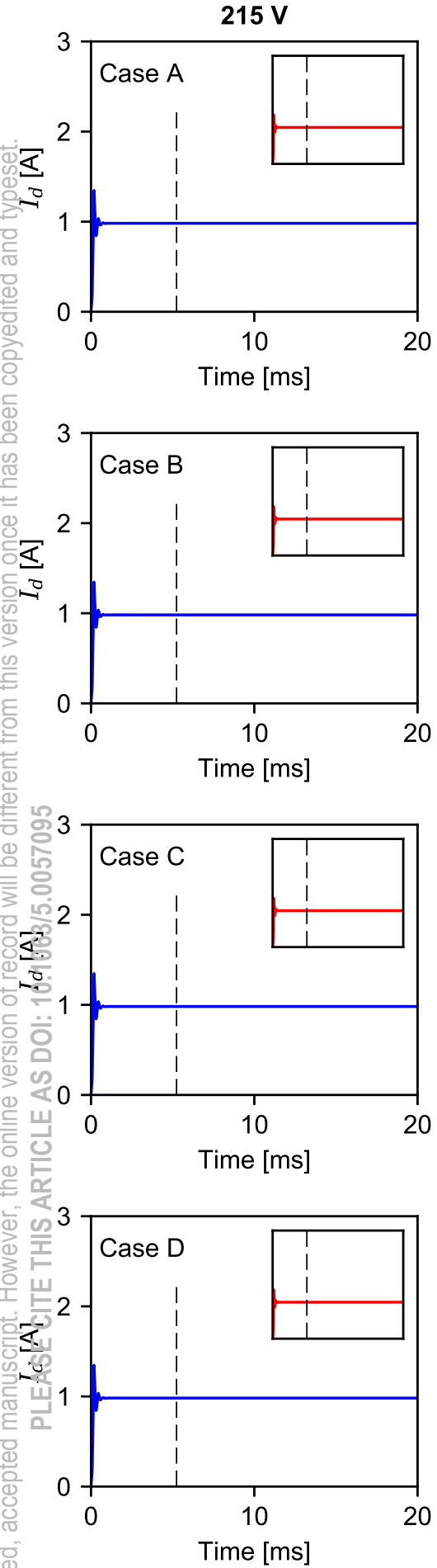

$250 \mathrm{~V}$
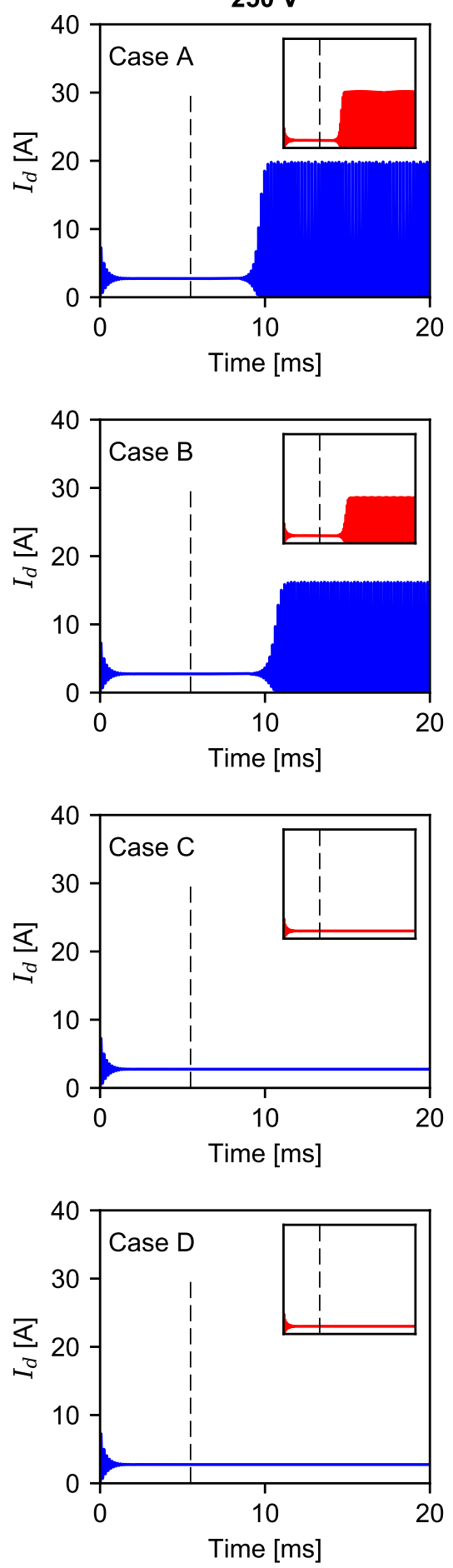

275 V
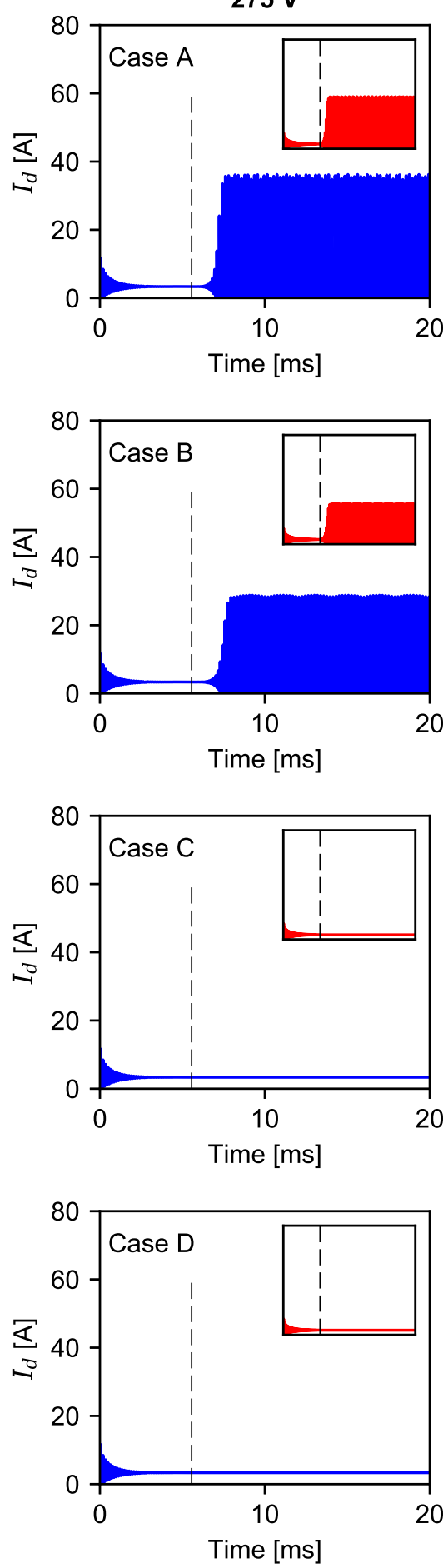

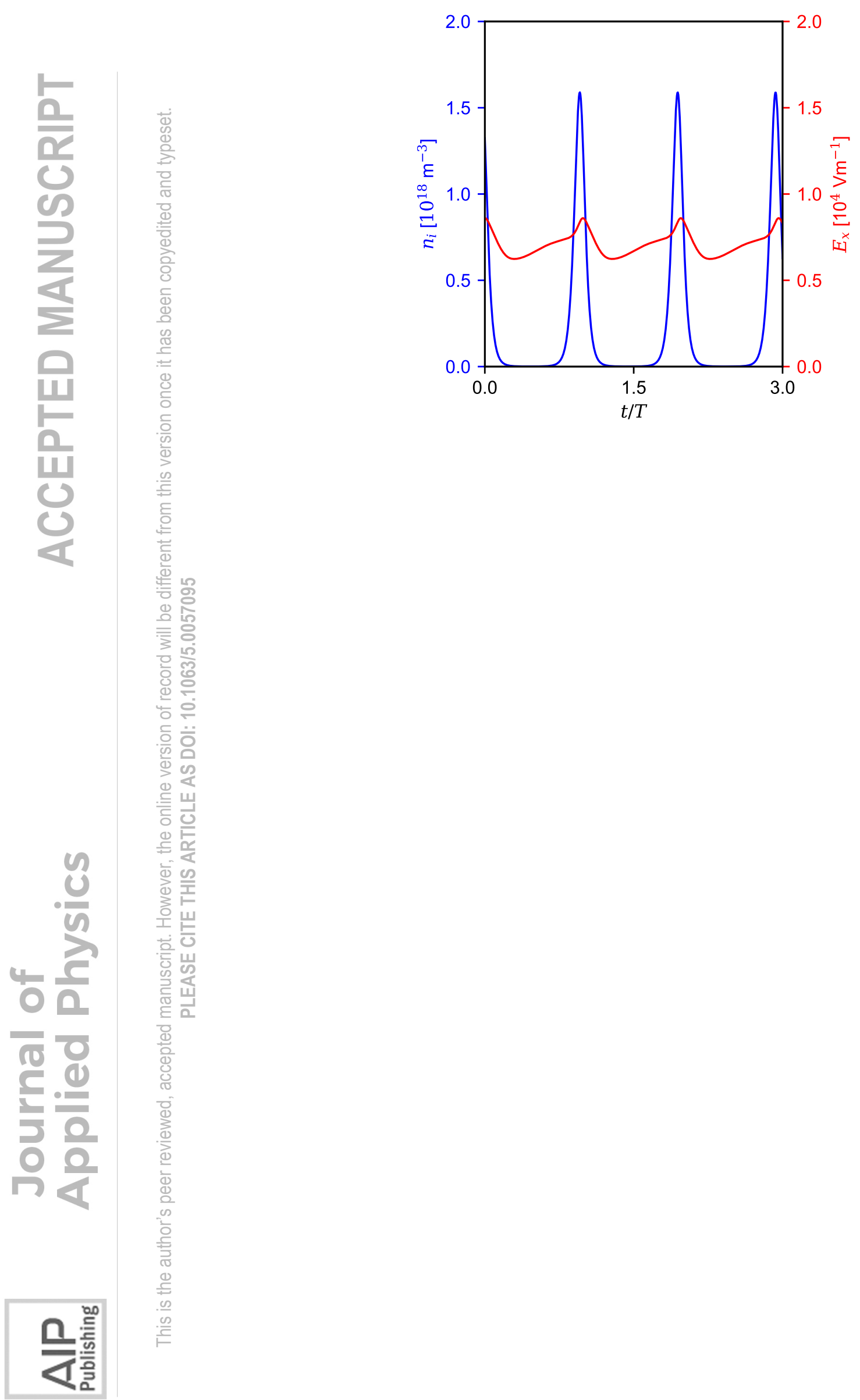

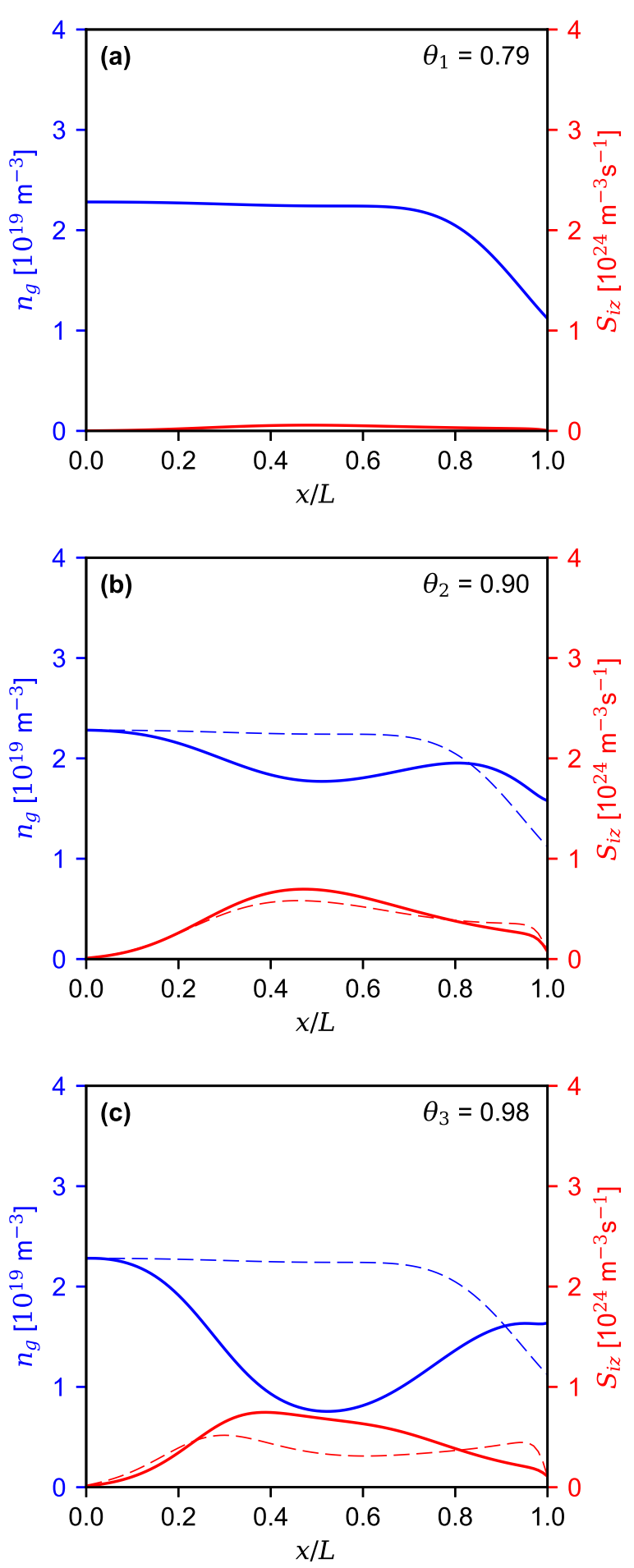
